\title{
Deficiency of autophagy protein Map1-LC3b mediates IL-17-dependent lung pathology during respiratory viral infection via ER stress-associated IL-1
}

\author{
M Reed ${ }^{1}$, SH Morris ${ }^{1}$, AB Owczarczyk ${ }^{1}$ and NW Lukacs ${ }^{1}$
}

While recent studies suggest that interleukin (IL)-1ß production is modulated by macroautophagy or sensors of endoplasmic reticulum (ER) stress upon pro-inflammatory insult, autophagy and IL-1ß production during viral infection has not been fully investigated. This was addressed using respiratory syncytial virus (RSV), which is associated with lung immunopathology, IL-1, and IL-17a secretion in severely infected patients. Mice deficient in the autophagy-associated protein Map1-LC3b (LC3b ${ }^{-/-}$) developed increased IL-17a-dependent lung pathology upon infection. RSV-infected $\mathrm{LC} \mathrm{b}^{-1-}$ dendritic cells (DCs) fail to upregulate autophagosome formation, secrete IL-1 $\beta$ and IL-6, and elicit IL-17a production from CD4 + Tcells. Bone marrow chimeras revealed that both structural and hematopoietic LC3b deficiency contribute to the development of IL-17a-dependent lung pathology in vivo. Further investigation revealed airway epithelium as the primary source of IL-1 $\beta$ during infection, whereas inhibition of the ER-stress sensor inositol-requiring protein-1 in primary airway epithelial cells reduced IL-1 $\beta$ production identifying a primary ER stress pathway. Finally, blockade of IL-1 receptor signaling in RSV-infected LC3b ${ }^{-1}$ mice abolished IL-17a-dependent lung pathology. These findings provide novel mechanistic insight into the contribution of autophagy- and ER stress-dependent cytokine production that initiate and maintain aberrant Th17 responses, while identifying IL-1 as a potential therapeutic target in the treatment of severe respiratory viral infections.

\section{INTRODUCTION}

Human respiratory syncytial virus (RSV) remains the leading cause of infant hospitalization in the United States, ${ }^{1,2}$ and is responsible for considerable morbidity among infants, the elderly, and individuals with chronic respiratory illnesses worldwide. ${ }^{3-5}$ Hospitalization with RSV as an infant is also highly correlated with the development of recurrent wheezing in childhood, suggesting lasting immune environment alteration in the lung post infection. ${ }^{6}$ The severity of lowerrespiratory tract RSV infection in otherwise healthy individuals is largely driven by an overexuberant immune response to the virus, and is characterized by bronchiolitis, epithelial cell sloughing, mucus hypersecretion, and infiltration of neutrophils into the airways. ${ }^{78}$ Elevated levels of the pro-inflammatory cytokines interleukin (IL)-1, IL-6, and IL-17a have recently been noted in respiratory aspirate samples from patients hospitalized with severe RSV infections, ${ }^{9-11}$ whereas experimental evidence suggests that IL-17a production drives mucus hypersecretion, neutrophil infiltration, and the suppression of CD8 + T-cell responses in the context of RSV infection. ${ }^{10,12,13}$ The mechanisms by which Th17 immune responses to RSV are initiated and maintained remain poorly understood.

Within the lung environment, resident dendritic cells (DCs) detect invading pathogens and mediate both innate and adaptive immune responses through cytokine secretion and antigen presentation to $\mathrm{T}$ cells. Classical induction of IL-17a production by murine $\mathrm{CD} 4+\mathrm{T}$ cells requires expression of the innate cytokines IL- 6 and transforming growth factor $\beta$ (TGF $\beta$ ) by antigen-presenting cells, whereas IL- 23 is

1Department of Pathology, University of Michigan Medical School, Ann Arbor, Michigan, USA. Correspondence: NW Lukacs (nlukacs@umich.edu) Received 20 August 2014; accepted 24 December 2014; published online 11 February 2015. doi:10.1038/mi.2015.3 
required for the expansion and survival of Th17 cells. ${ }^{14,15}$ In contrast, the induction of IL-17a secretion by human CD $4+\mathrm{T}$ cells requires IL- 1 and IL- 6 but not TGF $\beta,{ }^{16}$ whereas IL-1 receptor signaling is required for the production of IL-17a by both human and mouse CD $4+$ T cells. ${ }^{17}$ The production of IL23 by DCs in response to Toll-like receptor (TLR) ligands is further driven by IL- $1 \beta,{ }^{18}$ suggesting a critical role for IL-1 in the maintenance of Th17 responses.

The IL- 1 family proteins IL- $1 \beta$ and IL-18 are synthesized upon TLR activation in an inactive precursor state, and regulated via post-translational cleavage by assembled inflammasome complexes such as AIM2 and NLRP $3 .{ }^{19}$ The secretion of IL- $1 \beta$ and IL-18 by DCs and macrophages upon TLR activation is also negatively regulated by macroautophagy (autophagy), a homeostatic mechanism by which cytosolic constituents are enveloped in a double-walled membrane and delivered to lysosomes for degradation. Pro-IL-1 $\beta$ and proIL-18 are rapidly sequestered within autophagosomes upon the induction of autophagy, ${ }^{20}$ whereas ubiquitinated inflammasomes are bound by sequestosome-1/p62 and selectively targeted to nascent autophagosomes through p62 binding to membrane-bound Map-1LC3b (LC3b). ${ }^{21}$ It was recently reported that RSV infection activates the NLRP3 inflammasome and induces IL-1 $\beta$ secretion from human bronchial epithelial cells, ${ }^{22}$ as well as murine macrophages and epithelial cells. ${ }^{23}$ Our laboratory has found evidence supporting a critical role for autophagy in DCs by promoting activation, cytokine secretion, and antigen presentation upon RSV infection in vitro, ${ }^{24}$ whereas autophagy deficiency in DCs enhances RSV-induced pathology in vivo. ${ }^{25}$ However, the potential modulatory role of autophagy in controlling excessive inflammasome activation and IL-1 $\beta$ secretion during RSV infection is yet to be examined.

As RSV infects via a membrane fusion event that allows direct entry of its genetic material into the cytoplasm, activation of critical innate TLR responses relies on autophagy to transport viral pathogen associated molecular patterns and promotes appropriate antiviral responses. ${ }^{24}$ To investigate the potential role of autophagy in mitigating RSV-induced lung pathology in vivo, mice deficient in the critical autophagy protein LC3b $\left(\mathrm{LC} 3 \mathrm{~b}^{-1-}\right)$ were used. These mice have been previously reported to express greater NLRP3 inflammasome activation, IL-1 $\beta$ secretion, and increased susceptibility to experimental sepsis. ${ }^{26}$ Our results demonstrate that LC3b is critical to the regulation of Th17-induced lung pathology during RSV infection, as LC3b ${ }^{-1-}$ mice show greater mucus hypersecretion, lung neutrophil infiltration, and IL-17a production, which was ameliorated by IL-17a neutralization. These diseaseassociated pathologies were associated with increased IL- $1 \beta$ and IL- 6 production in the LC3b-deficient mice during RSV infection that were related to endoplasmic reticulum (ER) stress responses on the basis of specific pathway blockade. Importantly, blockade of IL-1 receptor signaling in vivo substantially reduced IL-17a secretion and immunopathology in the lungs of $\mathrm{LC} \mathrm{b}^{-1-}$ mice, suggesting a potential therapeutic approach to alleviating severe RSV-induced responses.

\section{RESULTS LC3b $^{-I-}$ mice develop increased IL-17a-dependent lung pathology upon RSV infection}

As development of severe RSV infection in humans is characterized by bronchiolitis, mucus hypersecretion, and neutrophil accumulation in the lungs [], these parameters were assessed in RSV-infected $\mathrm{LC}^{-1-}$ mice at 8 days post infection. Lung histological sections stained with hematoxylin and eosin revealed greater peribronchial edema and granulocyte infiltration into lungs of RSV-infected LC3b $\mathrm{b}^{-1-}$ mice, in comparison with wild-type (WT) littermate controls (Figure 1a). In addition, greater periodic acid-schiff-positive mucus staining was visible along the apical epithelial surface of the major airways of RSV-infected LC $3 \mathrm{~b}^{-1-}$ mice (Figure 1a), whereas mRNA transcripts for the mucus-associated genes muc5ac and gob5 were elevated in the lungs of $\mathrm{RSV}$-infected LC3 $\mathrm{b}^{-1-}$ mice (Figure 1b). In order to assess lung viral replication, quantitative PCR (qPCR) for mRNA transcripts encoding the RSV-G and -F proteins was performed on whole lung homogenates. This revealed increased viral transcripts in the lungs of infected LC $3 \mathrm{~b}^{-1-}$ mice, which suggests decreased viral clearance relative to WT littermates (Figure 1c). Finally, in agreement with our histological findings, significantly higher numbers of neutrophils were detected in the lungs of RSV-infected LC3b ${ }^{-1-}$ mice with flow cytometry (Figure 1d).

Experimental evidence has shown a causative role for T-cellassociated cytokines, most recently IL-17a, in the development of severe lung pathology during RSV infection. ${ }^{10}$ Whole-lung lysates from RSV-infected $\mathrm{LC}^{-1-} \mathrm{b}^{-1-}$ mice revealed greater IL-17a protein production in comparison with littermate controls (Figure 1e). Conversely, lung mRNA expression of interferon $\gamma$ (IFN $\gamma$ ) was significantly lower in comparison with littermate controls (Figure 1f). The presence of IL-1, IL-6, and TGF $\beta$ is required for the induction of mucosal Th17 responses in mice, ${ }^{15,17}$ and indeed, lung protein levels of IL- $1 \alpha$, IL- $1 \beta$, and IL-6 were elevated in whole-lung lysates from RSV-infected LC3 $\mathrm{b}^{-1-}$ mice (Figure 1g). In contrast, no differences were detected in lung mRNA expression of TGF $\beta$, whereas lung protein levels of the inflammasome-dependent cytokine IL-18 did not differ between LC3b ${ }^{-1-}$ mice and WT littermates (data not shown). Finally, mediastinal lymph node (MedLN) cell cultures were restimulated ex vivo with RSV and demonstrated that LC $3 \mathrm{~b}^{-1-}$ cultures secreted significantly greater amounts of IL-17a in comparison with WT cultures (Figure 1h).

Previous studies found that $\mathrm{CD} 4+\mathrm{T}$ cells are the predominant sources of IL-17a in the lungs of WT mice at 8 days post-RSV infection []. However, recent advances in other IL-17-dependent disease models indicate that $\gamma \delta$-T cell receptor-positive $\mathrm{T}$ cells and innate lymphoid cells produce large quantities of IL-17a in response to IL-1 receptor signaling. In order to determine which cell types were responsible for elevated IL-17a production in LC3 $\mathrm{b}^{-1-}$ mice, intracellular staining and flow cytometry were performed on lung digests and MedLN cells from RSV-infected LC $3 b^{-1-}$ mice. Whereas 
a
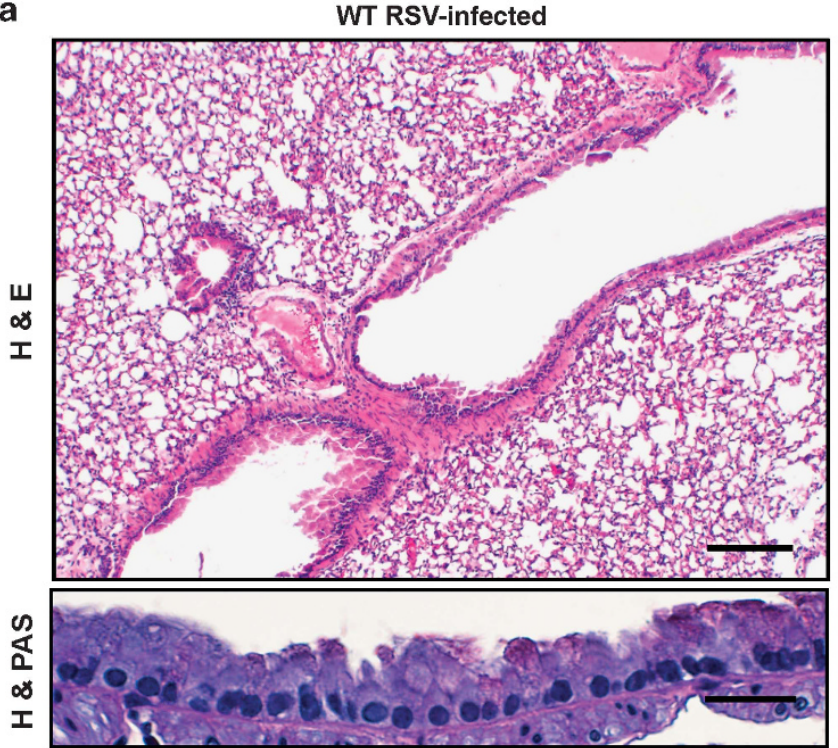
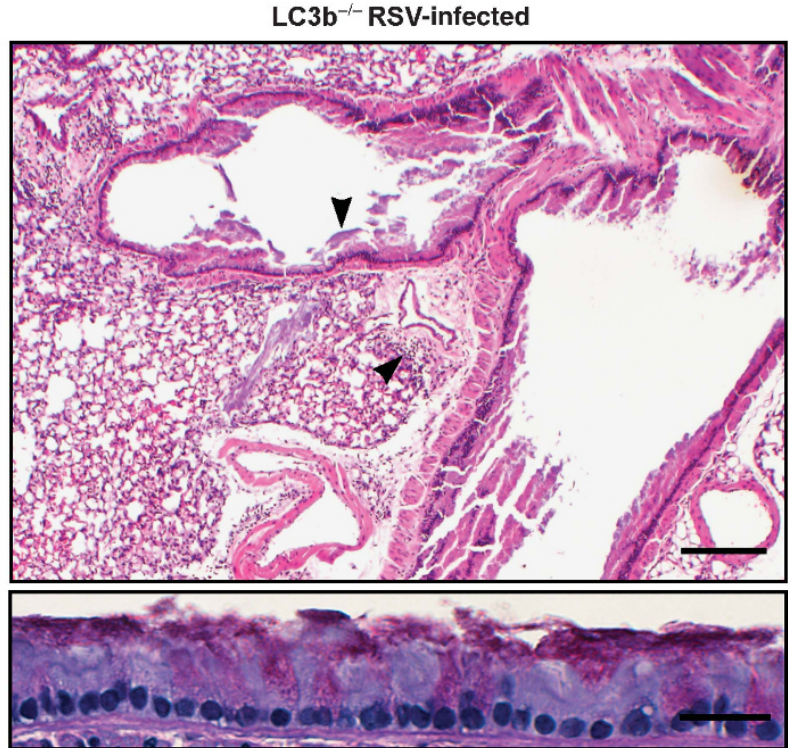

b

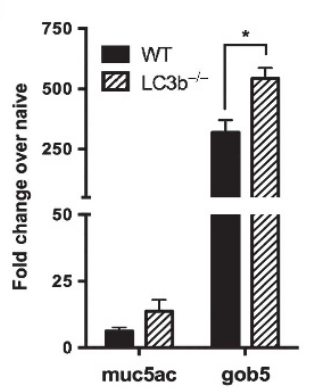

C

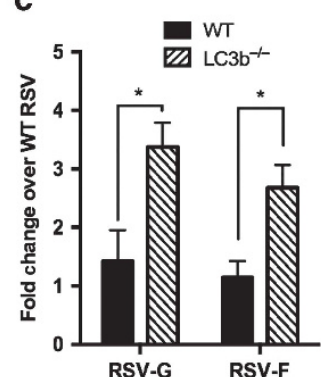

d

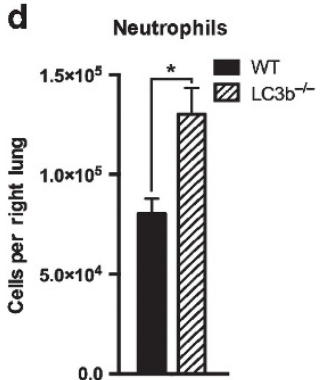

e

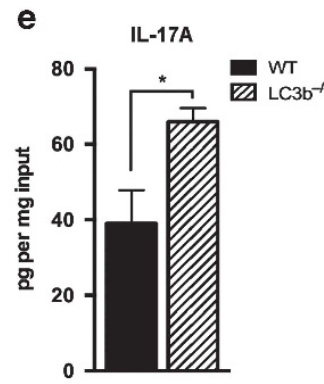

f f IFN $\gamma$

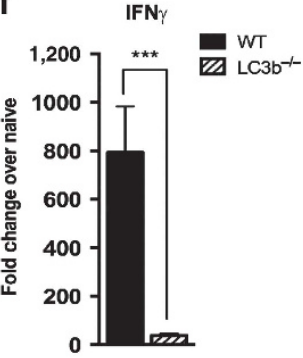

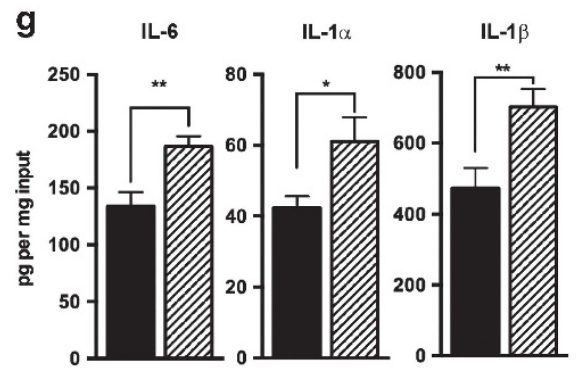
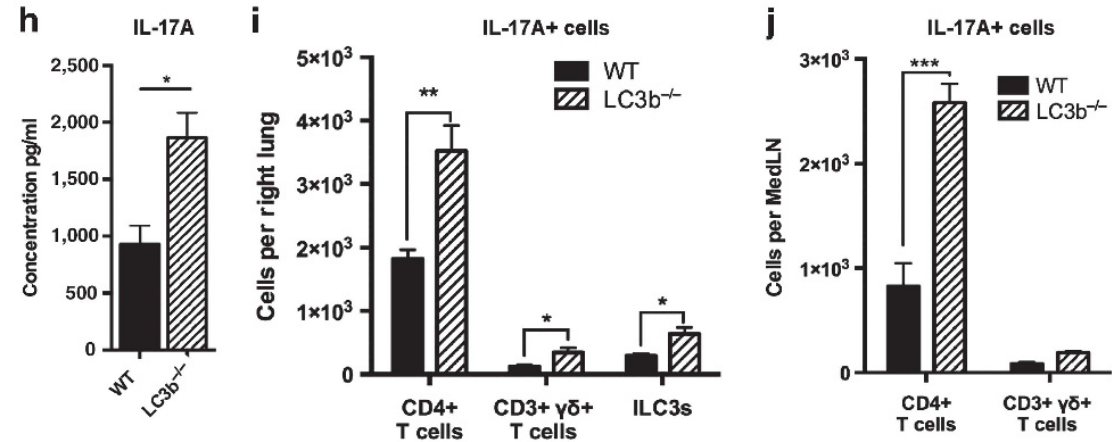

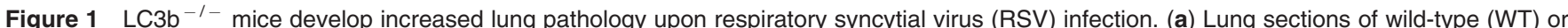
$\mathrm{LC} \mathrm{b}^{-1-}$ mice, 8 days post infection (DPI) with RSV, were stained with either hematoxylin and eosin (H \& E, upper panels) or periodic acid schiff and hematoxylin (H \& periodic acid-schiff (PAS), lower panels) to visualize mucus along the airway epithelium. Arrows indicate inflamed bronchiole (top) and perivascular granulocyte infiltration (bottom). (b) Lung mRNA expression of muc5ac and gob5 at 8 DPI was obtained using quantitative PCR (qPCR), compared with naive controls. (c) mRNA transcripts from lungs, encoding RSV-G and -F proteins, were measured using qPCR and normalized to values of WT RSV-infected lung samples. (d) Neutrophils in the right lungs of WT and LC3b ${ }^{-1}$ mice at 8 DPI were quantified using flow cytometry, following enzymatic digestion of the lungs. Total granulocytes were gated CD $11 b^{\text {hi }} \mathrm{CD}_{11} \mathrm{c}^{\mathrm{lo}} \mathrm{SSC}^{\text {hi }}$ Auto ${ }^{\text {lo }}$, with neutrophils gated Ly $6 \mathrm{G}^{\text {hi }}$ Ly6C ${ }^{+}$. (e) Interleukin (IL)$17 a$ concentrations in whole-lung lysates, normalized to protein input, were measured using the Bioplex assay. (f) Lung interferon $\gamma($ IFN $\gamma$ ) values were obtained using qPCR. Fold change values were calculated relative to naive control mice. (g) Concentrations of IL-6, IL-1 $\alpha$, and IL-1 $\beta$ were measured in whole-lung lysates using the Biopex assay, normalized to protein input. (h) Culture supernatant concentrations of IL-17a in RSV-restimulated lymph node cultures was measured at $48 \mathrm{~h}$ using the Bioplex assay. (i) Single-cell suspensions prepared from enzymatically digested lungs were treated with Golgi Stop, stimulated in culture with $10 \mathrm{ng} / \mathrm{ml} \mathrm{PMA}$ and $1 \mu \mathrm{g} / \mathrm{ml}$ lonomycin for $5 \mathrm{~h}$, followed by surface/intracellular staining and analysis with flow cytometry. $\mathrm{CD} 4+\mathrm{T}$ cells were gated CD $45^{+} \mathrm{CD}^{+} \mathrm{CD} 4^{+} \gamma \delta^{-}, \gamma \delta \mathrm{T}$ cells gated CD $45^{+} \mathrm{CD}^{+} \gamma \delta^{+}$, and innate lymphoid cells (ILCs) gated CD45 ${ }^{+}$Lineage $^{-}$ Thy $1.2^{+} \mathrm{ROR} \mathrm{t}^{+}$. (j) Single-cell preparations from mediastinal lymph nodes (MedLNs) were stained and gated as in (i). Results are representative of at least two independent experiments, $n \geq 4$ mice per group. ${ }^{\star} P \leq 0.05,{ }^{\star \star} P \leq 0.01,{ }^{* \star \star} P \leq 0.001$. Bars $=500 \mu \mathrm{m}$ for upper panels, $20 \mu \mathrm{m}$ for lower panels.

the numbers of $\gamma \delta$-receptor-positive $\mathrm{T}$ cells and innate lymphoid cells positive for IL-17a were increased in the lungs of $\mathrm{LC} 3 \mathrm{~b}^{-/-}$mice in comparison with WT littermates, the majority of IL-17a-positive cells were CD4 $+\mathrm{T}$ cells (Figure 1i). Significantly greater numbers of IL-17a-positive $\mathrm{CD} 4+\mathrm{T}$ cells were also detected in MedLNs of $\mathrm{LC} \mathrm{b}^{-1-}$ mice 


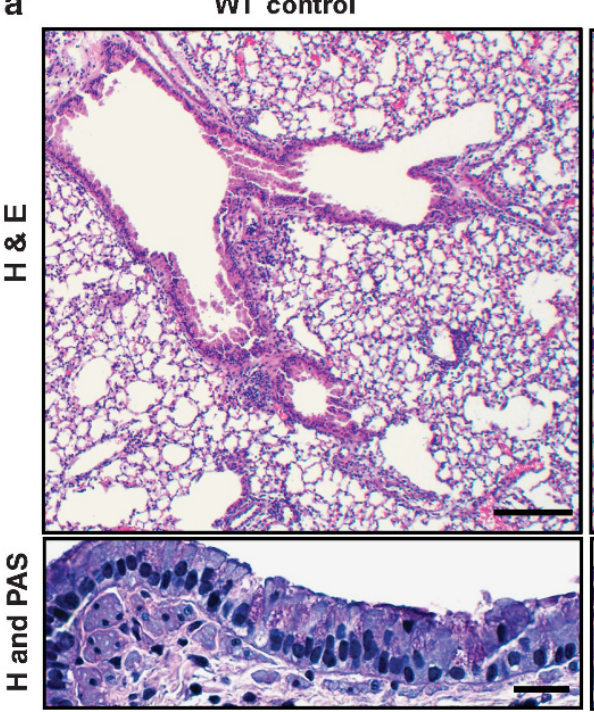

LC3 $b^{-1-}+$ Cont Ab

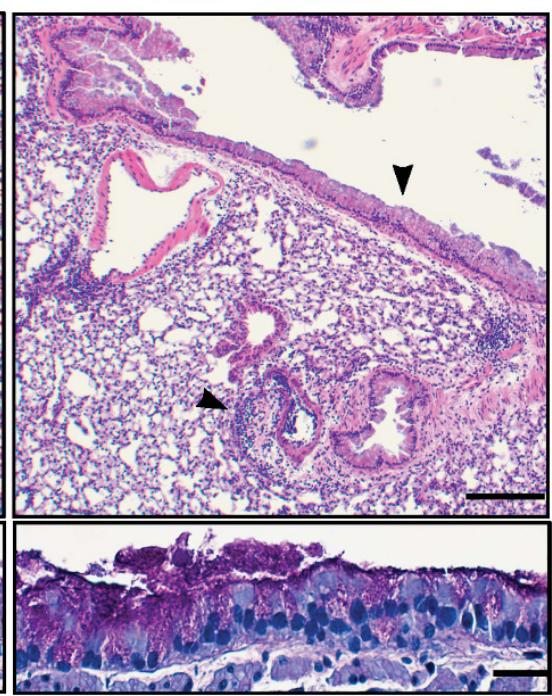

LC3 $b^{-1-}+\alpha-$ IL-17A Ab

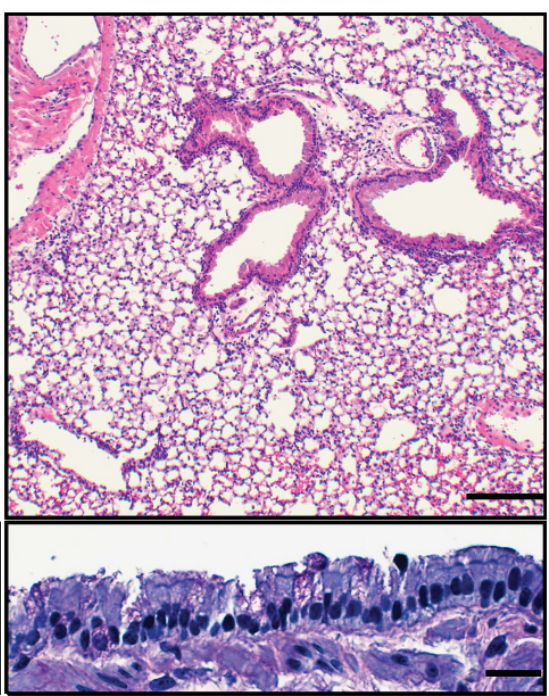

b

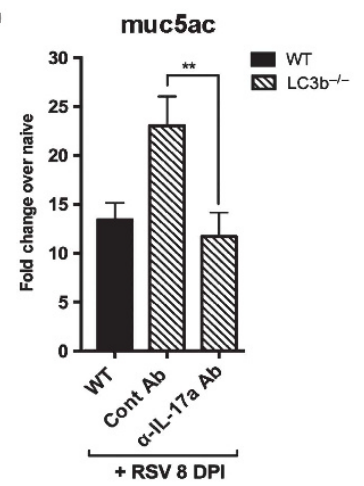

c

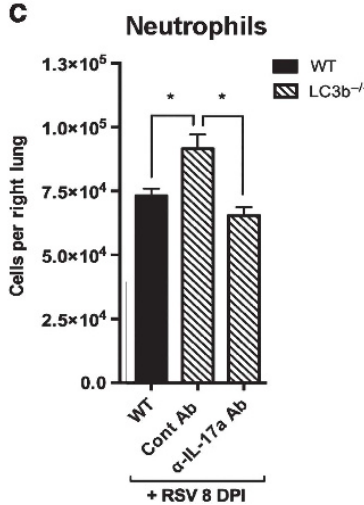

d

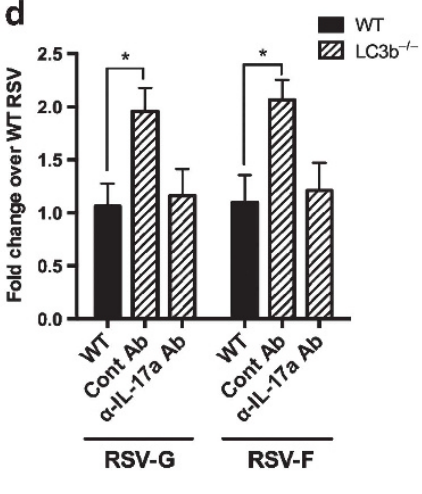

e

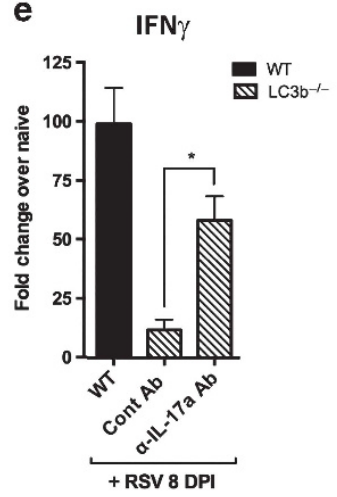

Figure 2 Increased respiratory syncytial virus (RSV)-induced lung pathology in LC3b ${ }^{-1}$ mice is interleukin (IL)-17a-dependent. LC3b ${ }^{-1}$ mice were treated with $2.5 \mathrm{mg}$ nonspecific control antibody, or polyclonal antibody to mouse IL-17a, immediately before RSV infection and on days 2 , 4, and 6 post infection. (a) Lungs were harvested at $8 \mathrm{DPI}$, sectioned, and stained with hematoxylin and eosin (H \& E) or hematoxylin and periodic acid schiff (H and periodic acid-schiff (PAS)) to visualize mucus. Arrows indicate inflamed bronchiole (top) and perivascular granulocyte infiltration (bottom). (b) Lung expression of the mucin gene muc5ac was measured at 8 DPI by quantitative PCR (qPCR), relative to naive controls. (c) Neutrophils were quantified by flow cytometric analysis of enzymatically digested lungs, $8 \mathrm{DPI}$. Neutrophils were gated as indicated in Figure 1. (d) Lung mRNA expression encoding RSV-G and RSV-F was assessed at 8 DPI, relative to RSV-infected wild-type (WT). (e) Lung interferon $\gamma$ (IFN $\gamma$ ) expression was measured at 8 DPI with qPCR. Results are representative of three independent experiments, $n \geq 4$ mice per group. ${ }^{\star} P \leq 0.05$, ${ }^{\star *} P \leq 0.01,{ }^{* \star *} P \leq 0.001$. Bars $=500 \mu \mathrm{m}$ on upper panels, $20 \mu \mathrm{m}$ on lower panels.

(Figure 1j), suggesting that increased production of IL-17a by $\mathrm{CD} 4+\mathrm{T}$ cells are primarily responsible for elevated IL-17a levels in both the lungs and MedLNs of RSV-infected $\mathrm{LC} 3 \mathrm{~b}^{-1-}$ mice.

Previous studies conducted by our laboratory found that IL-17a production contributes to lung inflammation, excessive mucus production, and the suppression of IFN $\gamma$ production by CD8 + T cells during RSV infection. ${ }^{10,27}$ To assess whether the increased lung pathology in RSV-infected LC3 $\mathrm{b}^{-1-}$ mice was IL-17a-dependent, $\mathrm{LC}^{-1-}$ mice were treated with neutralizing antibodies to IL-17a. In comparison with control antibody treatment, administration of anti-IL-17a antibodies to RSV-infected LC3b ${ }^{-1-}$ mice substantially decreased visible lung inflammation and leukocyte infiltration at 8 days post infection (Figure 2a). Neutralization of IL-17a significantly decreased periodic acid-schiff-positive mucin staining along the bronchial epithelium, which was verified by decreased muc5ac expression in lungs of anti-IL-17a-treated LC3 $\mathrm{b}^{-1-}$ mice at 8 days post infection (Figure $2 \mathbf{a}$ and $\mathbf{b}$ ). Neutralization of IL-17a also reduced the neutrophil accumulation in the LC3 - / - mice (Figure 2c). Remarkably, neutralization of IL-17a rescued viral clearance in $\mathrm{LC} 3 \mathrm{~b}-/-$ mice, as anti-IL-17a-treated LC3b-/ mice showed lung viral mRNA levels equivalent to WT controls (Figure 2d). Lung IFN $\gamma$ expression was also significantly higher in comparison with control-antibody-treated LC3b-/- mice corresponding to a more effective cellular immune response (Figure 2e). These results collectively suggest that the development of increased lung pathology in $\mathrm{LC}^{-1-}$ mice is IL-17a-dependent. 


\section{Altered autophagy, innate cytokine production, and CD4 + T-cell cytokine elicitation by LC3b ${ }^{-1-}$ CD11b + DCs in response to RSV infection}

Recent work identified a requirement for $\mathrm{CD} 11 \mathrm{c}^{\text {high }} \mathrm{CD} 11 \mathrm{~b}+$ tissue-resident DCs in the induction of mucosal Th17 immune responses. ${ }^{28,29}$ As pharmacological blockade of autophagy increases IL-23 secretion by DCs and macrophages in response to TLR agonists, ${ }^{18}$ the ability of LC $3 b^{-1-}$ DCs to upregulate autophagosome formation and secrete innate cytokines in response to RSV was investigated. Confocal microscopy revealed robust formation of ATG5 + autophagosomes by WT bone marrow-derived DCs (BMDCs) in response to RSV infection (Figure 3a). In contrast, $\mathrm{LC} \mathrm{b}^{-1-}$ BMDCs failed to increase the formation of ATG5 + puncta in response to RSV (Figure 3a). In agreement with these observations, levels of the sequesteosome protein p62 decreased in whole-cell lysates of WT BMDCs by $8 \mathrm{~h}$ post infection, whereas $\mathrm{LC} \mathrm{b}^{-1-}$ BMDCs continued to accumulate p62 over a 24-h period (Figure $3 \mathbf{b}$ ). Analysis of cytokine secretion by RSV-infected BMDCs revealed significantly higher production of IL-1 $\beta$ and IL-6 by $\mathrm{LC} \mathrm{b}^{-1-}$ BMDCs (Figure 3c). LC3b $\mathrm{b}^{-1-}$ BMDCs expressed less of the IL-12-specific subunit IL-12p35 and the IL-12/IL-23 common subunit IL-12p40 with qPCR, in comparison with WT BMDCs (Figure 3d). Conversely, RSVinfected LC3b ${ }^{-1-}$ BMDC significantly upregulated IL-23p19 expression in comparison with WT BMDCs (Figure 3d). These findings were confirmed using $\mathrm{CD} 11 \mathrm{c}^{\text {high }} \mathrm{CD} 11 \mathrm{~b}^{+}$lamina propria DCs $\left(\mathrm{CD} 11 \mathrm{~b}^{+} \mathrm{LP}\right.$ DCs), flow-sorted from lungs of naive $\mathrm{WT}$ and $\mathrm{LC}_{3} \mathrm{~b}^{-1-}$ mice that were infected ex vivo with RSV. LC $3 b^{-1-}$ CD $11 b^{+}$LP DCs secreted greater amounts of IL- $1 \beta$ and IL- 6 upon RSV infection, whereas IL-12p35 and IL-12p40 mRNA expression levels were significantly reduced in comparison with WT controls (Figure $3 \mathbf{e}$ and $\mathbf{f}$ ). In order to assess whether $\mathrm{LC} 3 \mathrm{~b}^{-1-}$ DCs preferentially elicit increased IL-17a secretion from CD4 $+\mathrm{T}$ cells in response to RSV infection, BMDCs were co-cultured with purified CD4 $+\mathrm{T}$ cells derived from MedLNs of RSV-infected WT mice. CD4 + MedLN T cells secreted significantly greater quantities of IL$17 \mathrm{a}$ in co-culture with RSV-infected LC $3 b^{-1-}$ BMDCs, in comparison with T cells co-cultured with infected WT BMDCs (Figure 3g). To further investigate DC elicitation of CD4+ T-cell IL-17a secretion as a consequence of autophagy deficiency, purified $\mathrm{CD} 4+$ splenic OT-II $\mathrm{T}$ cells were co-cultured with BMDCs pulsed with the ovalbumin protein. These latter studies were conducted in the presence or absence of RSV in order to provide an autophagic stimulus, as ovalbumin treatment alone does not induce autophagy. Whereas co-cultures in which WT and LC3b ${ }^{-1-}$ DCs were treated with ovalbumin alone produced similar quantities of IL$17 \mathrm{a}$, cultures in which $\mathrm{LC}^{-1-}$ DCs were concurrently infected with RSV elicited significantly greater IL-17a production from the CD4+ OT-II T cells (Figure $3 \mathbf{h}$ ). These findings were confirmed using $\mathrm{CD} 11 \mathrm{~b}^{+} \mathrm{LP}$ DCs isolated from lungs of naive WT and $\mathrm{LC}^{\mathrm{C}} \mathrm{b}^{-1-}$ mice, with RSVinfected and ovalbumin-pulsed $\mathrm{LC} \mathrm{b}^{-1-} \mathrm{CD} 11 \mathrm{~b}^{+} \mathrm{LP}$ DCs eliciting significantly higher quantities of IL-17a from co-cultured OT-II T cells (Figure 3i). These results support impaired autophagy and altered innate cytokine production by $\mathrm{LC} \mathrm{b}^{-1-}$ DCs as primary mechanisms in the initiation of Th17-skewed CD4 + T-cell responses to RSV infection.

\section{Non-immune cell deficiency in LC3b augments Th17- associated RSV pathology through increased IL-1 secretion by airway epithelial cells}

Results thus far indicated that $\mathrm{LC} \mathrm{b}^{-1-} \mathrm{CD} 11 \mathrm{~b}+$ DCs elicit increased IL-17a production from CD4 $+\mathrm{T}$ cells in response to RSV. As LC3b expression in epithelial cells is an important modulator of lung pathology upon exposure to cigarette smoke $^{30}$ or hyperoxia, ${ }^{31}$ bone marrow chimeric mice were created to assess relative contributions of structural and hematopoietic cells to observed RSV-induced lung pathology. Histologic examination of lung tissue 8 days post infection revealed that $\mathrm{WT}$ mice reconstituted with $\mathrm{LC} \mathrm{b}^{-1-}$ bone marrow experienced significant increases in lung inflammation, infiltration of neutrophils, and IL-17a production in comparison with fully WT chimeric animals (Figure 4a-c), suggesting that hematopoietic LC3b deficiency is sufficient to induce increased IL-17 production and neutrophil infiltration in response to RSV in vivo. Interestingly, reconstitution of $\mathrm{LC} \mathrm{b}^{-1-}$ mice with WT bone marrow produced an augmented inflammatory response, as significantly higher numbers of neutrophils were found in the lungs in comparison with all other treatment groups (Figure $4 \mathbf{b}$ ). These PMN accumulation data correlated well with the analysis that revealed augmented production of IL-17a in lung tissues and restimulated MedLN cultures (Figure $\mathbf{4 c}$ and d), with the WT to LC $3 \mathrm{~b}^{-1-}$ chimera having the most significant effect. Lung expression of IFN $\gamma$ was inversely correlated with IL-17a production (Figure 4e), whereas IL-6 expression was associated with structural LC3b deficiency (Figure 4f). Finally, investigation of innate cytokine concentrations in the lungs of chimeric mice revealed increased IL- $1 \alpha$ and IL-1 $\beta$ in lung lysates of RSV-infected LC3 ${ }^{-1-}$ chimeric mice, independent of WT or LC3 $\mathrm{b}^{-1-}$ bone marrow reconstitution (Figure 4d), implicating structural cells as the primary source of these cytokines in the lungs. These findings suggest that, whereas $\mathrm{LC} \mathrm{b}^{-1-}$ bone marrow is sufficient for the development of increased Th17-dependent lung pathology in response to RSV, $\mathrm{LC} \mathrm{b}^{-1-}$ lung structural cells contribute to the development of pathology through increased secretion of innate cytokines.

\section{Airway epithelial cells deficient in LC3b have enhanced inflammasome activation and increased cytokine production due to ER Stress}

To further examine innate cytokine production in response to RSV, fluorescent immunohistochemistry was used to examine IL-1 $\beta$ protein expression in lung sections of infected mice. This revealed greater staining intensity in lungs of RSV-infected $\mathrm{LC} \mathrm{b}^{-1-}$ mice, with the vast majority of IL- $1 \beta$-positive cells co-staining for the epithelial marker E-Cadherin (Figure 5a). To verify these findings, primary airway epithelial cell (AEC) cultures were derived from lungs of naive mice and infected with RSV in vitro. In agreement, $\mathrm{LC} \mathrm{b}^{-1-} \mathrm{AEC}$ cultures 


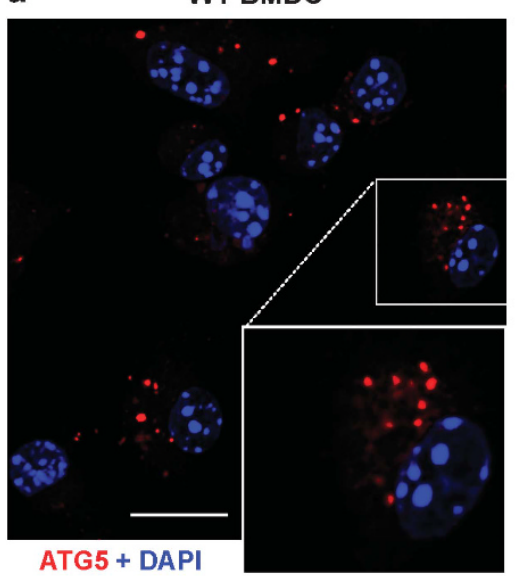

LC $3 b^{-1-}$ BMDC

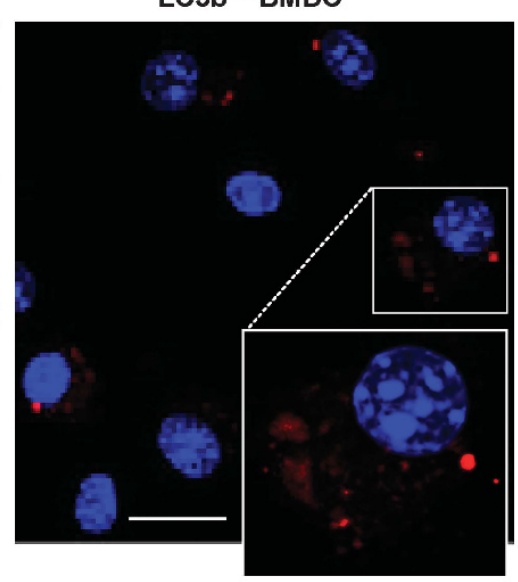

b
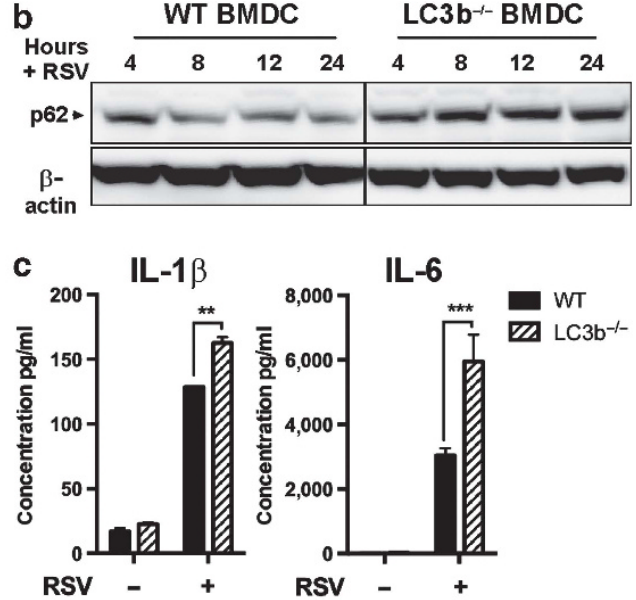

IL-1 $\beta$

IL-6

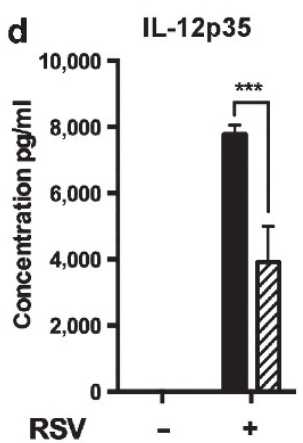

f

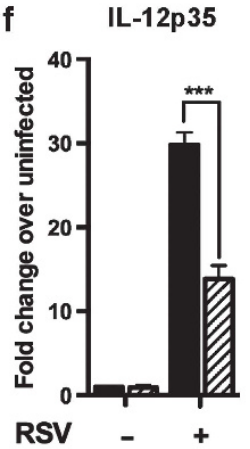

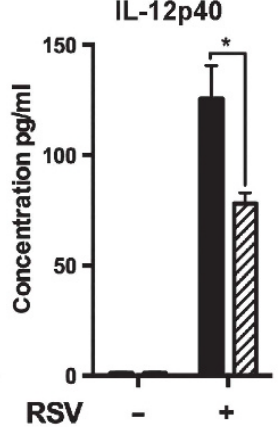

IL-12p40

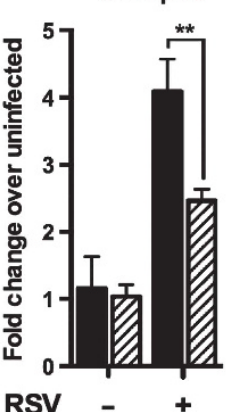

IL-23p19

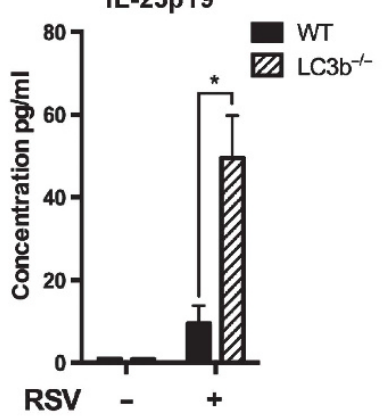

e
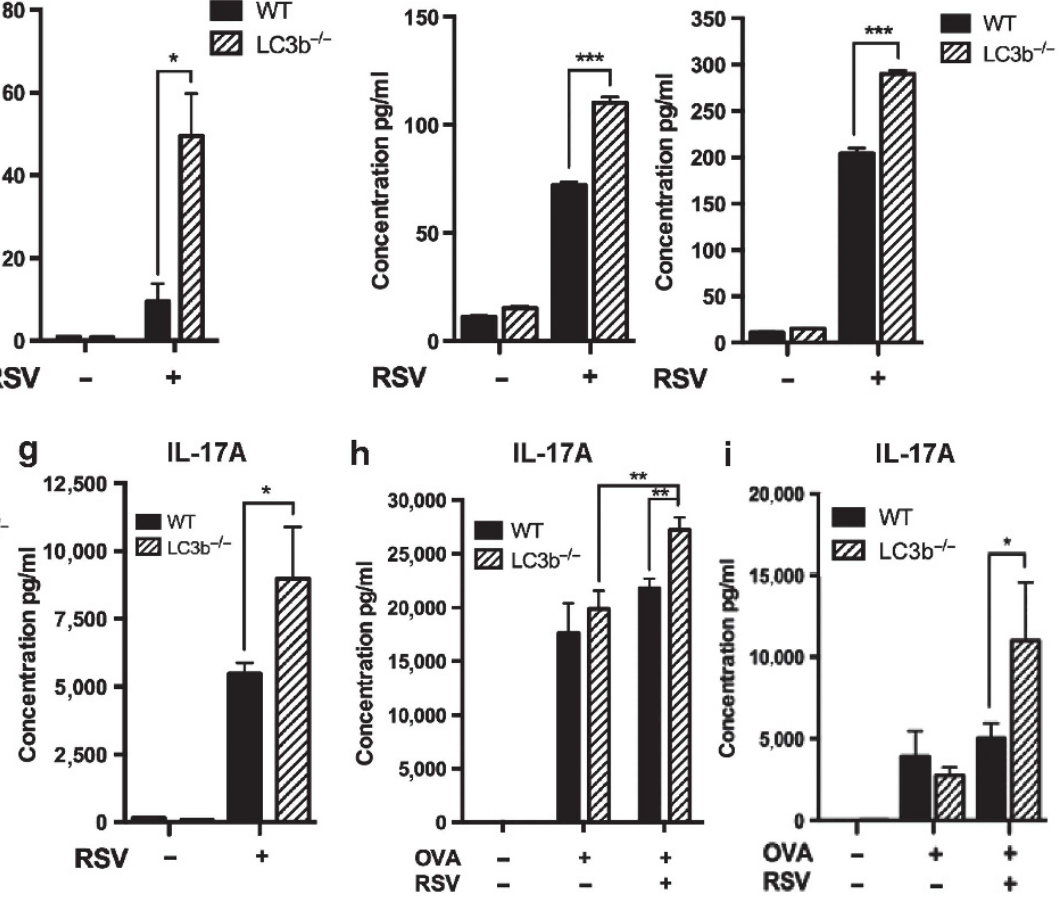

Figure 3 Altered autophagic flux, innate cytokine production, and elicitation of CD4 + T cell interleukin (IL)-17a production by LC3b ${ }^{-1-}$ dendritic cells (DCs) in response to respiratory syncytial virus (RSV). (a) Bone marrow-derived DCs (BMDCs) were cultured from naive wild-type (WT) or LC3b ${ }^{-1-}$ mice, and then infected for $4 \mathrm{~h}$ with RSV. Cells were fixed, stained with 4',6-diamidino-2-phenylindole (DAPI) and fluorescent antibody to ATG5, and imaged using confocal microscopy. (b) BMDCs were cultured and infected with RSV, followed by harvest at 4, 8, 12, and $24 \mathrm{~h}$ post infection. Whole-cell lysates were run on SDS-PAGE gels, followed by membrane transfer and immunoblot for p62 or $\beta$-actin as indicated. (c) Cell supernatants from BMDCs infected with 1:1 multiplicity of infection (MOI) RSV for $48 \mathrm{~h}$, analyzed using the Bioplex assay. (d) BMDCs were infected as above, harvested at $24 \mathrm{~h}$ post infection, and gene expression analyzed with quantitative PCR (qPCR). (e) Lung-resident CD11 $\mathrm{c}^{\text {hi }}$ Auto $^{\text {lo }} \mathrm{CD}_{11 b^{+}}$lamina propria DCs (CD11b ${ }^{+} \mathrm{LP}$ DCs) were flow-sorted from enzymatically digested lungs of naive WT and LC3b ${ }^{-1}$ mice. Cells were infected ex vivo with $1: 1 \mathrm{MOI}$ RSV, and cell supernatants harvested at $48 \mathrm{~h}$ post infection using the Bioplex assay. (f) Lung-resident CD11b ${ }^{+}$LP DCs were isolated and infected with RSV as previously described, followed by gene expression analysis using qPCR at $24 \mathrm{~h}$ post infection. (g) CD4 + T cells were purified from mediastinal lymph nodes of RSV-infected C57BI/6 mice, 8 days post infection. Cells were plated at a 10:1 ratio over WT or LC3b ${ }^{-1-}$ BMDCs and infected $2 \mathrm{~h}$ previously with

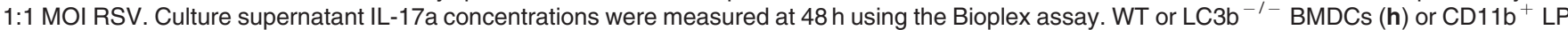
DCs (i) were pulsed with whole ovalbumin protein in the presence or absence of 1:1 MOI RSV, followed by the addition of $10: 1$ purified splenic OT-II CD4 + T cells. Culture supernatant IL-17a concentrations were measured at $48 \mathrm{~h}$ using the Bioplex assay. All results are representative of at least three independent experiments. ${ }^{*} P \leq 0.05,{ }^{* *} P \leq 0.01,{ }^{* * *} P \leq 0.001$. Bars $=10 \mu \mathrm{m}$.

upregulated IL-1 $\beta$ and IL-6 mRNA and secreted greater quantities of protein upon RSV infection, in comparison with WT cultures (Figure $5 \mathbf{b}$ and $\mathbf{c}$ ). Whereas IL- $1 \alpha$ mRNA was upregulated in RSV-infected LC3 ${ }^{-/-}$AECs (Figure 5b),
IL- $1 \propto$ was not detected in culture supernatants, possibly because of a lack of cell lysis in culture (data not shown). As $\mathrm{LC} 3 \mathrm{~b}^{-/-}$macrophages were also reported to secrete greater quantities of IL-1 $\beta$ in response to inflammasome-activating 
a
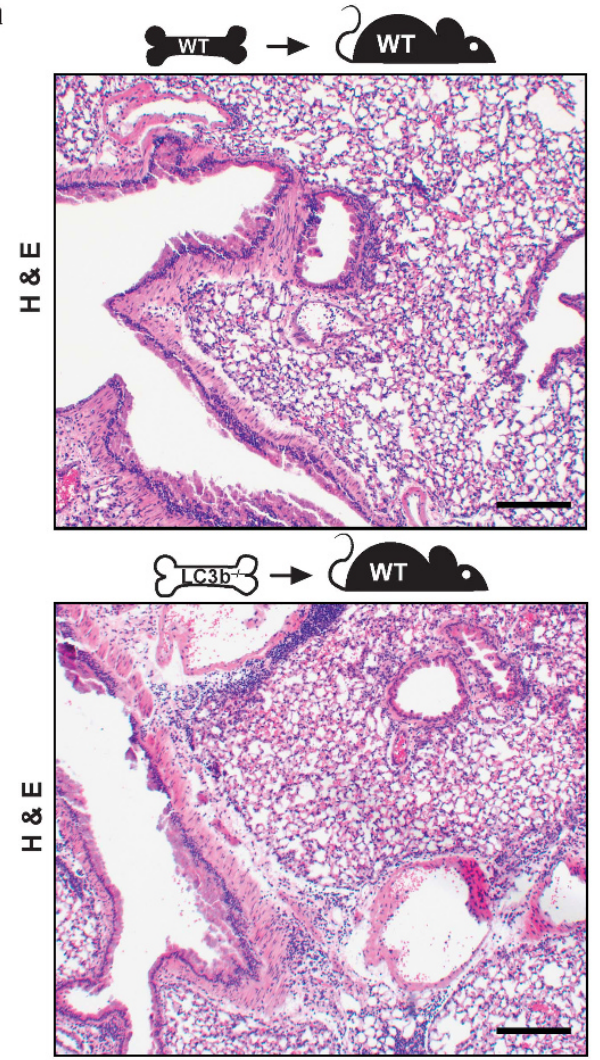
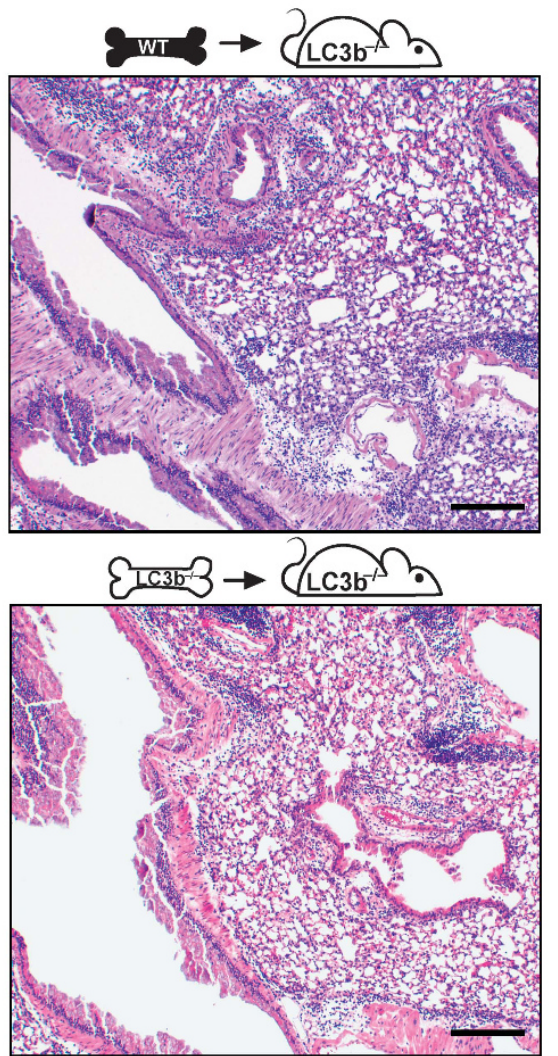

b

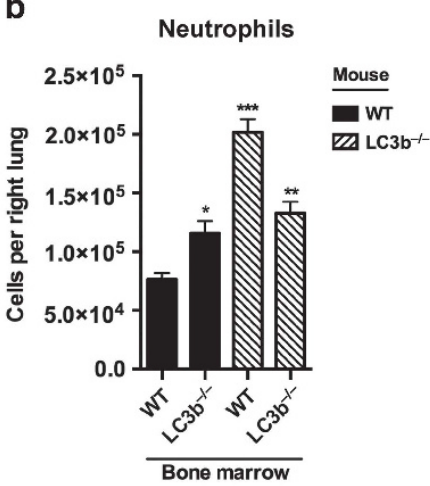

c

IL-17A

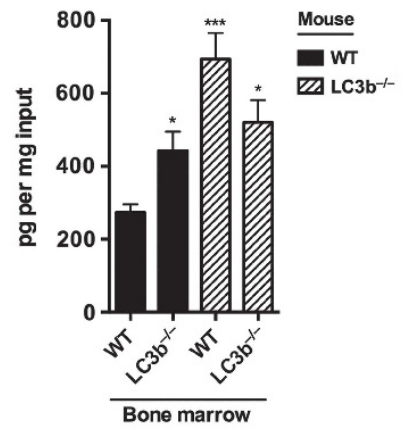

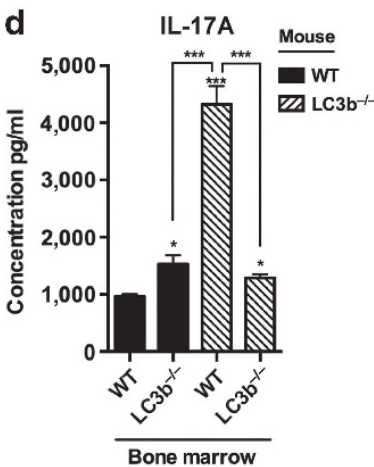
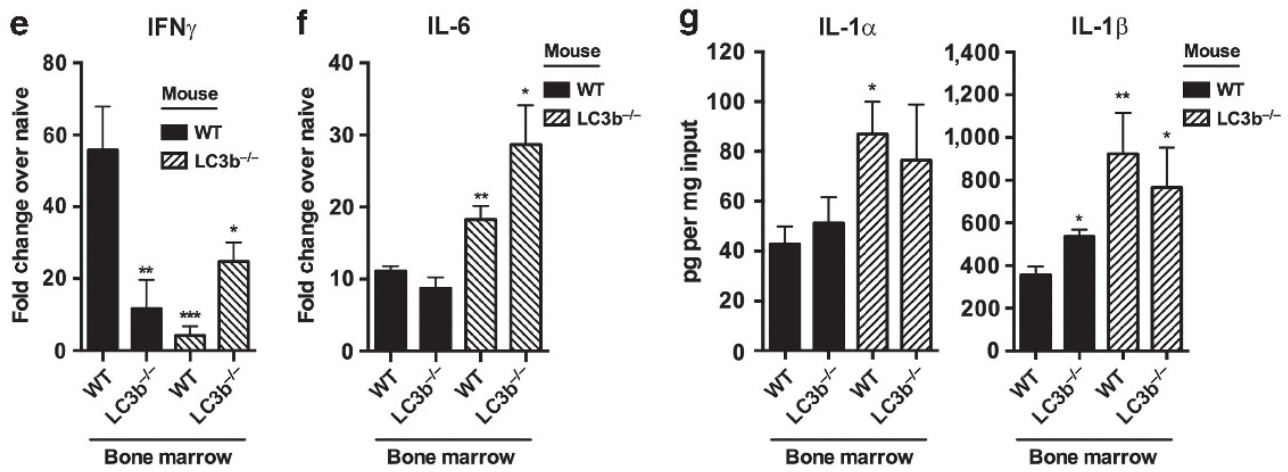

Figure 4 Both structural and hematopoietic LC3b deficiency contribute to increased lung pathology during respiratory syncytial virus (RSV) infection. Wild-type (WT) and LC3b $b^{-1-}$ mice were lethally irradiated and reconstituted with whole bone marrow from WT or LC $3 b^{-1-}$ donors, as indicated. Chimeric mice were infected with RSV and killed 8 days post infection. (a) Lung sections were stained with hematoxylin and eosin (H \& E) to visualize inflammation. (b) Neutrophils were quantified from enzymatically digested lungs with flow cytometry. Neutrophils were gated as indicated in Figure 1. (c) Concentrations of interleukin (IL)-17a in whole-lung lysates, normalized to protein input, were measured using the Bioplex assay. (d) IL-17a concentrations in RSV-restimulated mediastinal lymph node (MedLN) cultures were measured at $48 \mathrm{~h}$ using the Bioplex assay. Lung mRNA expression of interferon $\gamma$ (IFN $\gamma ; \mathbf{e}$ ) and IL-6 (f) were measured with quantitative PCR (qPCR). (g) Concentrations of IL-1 $\alpha$ and IL-1 $\beta$ in whole-lung lysates, normalized to protein input, were measured using the Bioplex assay. Results are representative of three independent experiments, $n \geq 4$ mice per group. ${ }^{\star} P \leq 0.05$, ${ }^{* *} P \leq 0.01,{ }^{* *} P \leq 0.001$. Bars $=500 \mu \mathrm{m}$.

stimuli, ${ }^{26}$ the expression of IL- $1 \beta$ by lung-infiltrating immune cells was examined with flow cytometry. This revealed greater intensity of IL- $1 \beta$ staining in LC3 $\mathrm{b}^{-1-}$ lung macrophages in response to RSV infection (Figure 5d), suggesting that these cells may also contribute to elevated IL- $1 \beta$ levels in lungs of

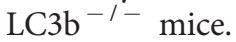

RSV infection is a known inducer of ER stress and NLRP3 inflammasome activation in bronchial epithelial cells. ${ }^{22,32}$ As prolonged ER stress reportedly triggers NLRP3 inflammasome activation, ${ }^{33}$ the expression levels of several genes activated during ER stress were examined in RSV-infected AEC cultures. In agreement with the previous report of signaling through the ER stress-sensing receptor inositol-requiring protein-1 (IRE1) $\alpha$ upon RSV infection, ${ }^{32}$ mRNA expression levels of Edem-1 and cleaved XBP-1 (XBP-1s) were significantly elevated in RSV-infected $\mathrm{LC} \mathrm{b}^{-1-}$ AEC cultures at $12 \mathrm{~h}$ post infection (Figure 5e). Furthermore, pre-treatment of LC3b ${ }^{-1-}$ AECs with the IRE- $1 \alpha$ inhibitor 3,5-dibromosalicylaldehyde reduced 
the secretion of IL- $1 \beta$ and IL- 6 to levels comparable to WT AECs (Figure 5f). Finally, in order to assess the contribution of ER stress to inflammasome activation during RSV infection, expression of the active $\mathrm{p} 10$ subunit of caspase- 1 was measured in primary AEC cultures infected with RSV. Confocal imaging and fluorescence quantification of caspase-1 p10 antibody staining revealed increased signal in $\mathrm{LC}^{-1-} \mathrm{b}^{-1}$ AECs $12 \mathrm{~h}$ post treatment with RSV, in comparison with WT control cultures (Figure 5g). Pre-treatment of $\mathrm{LC} \mathrm{b}^{-1-}$ AECs with 3,5dibromosalicylaldehyde abolished this difference (Figure 5g). Collectively, these results suggest that elevated RSV-induced production of IL- $1 \beta$ and IL- 6 by the $2 \mathrm{LC} 3 \mathrm{~b}^{-/-}$airway a

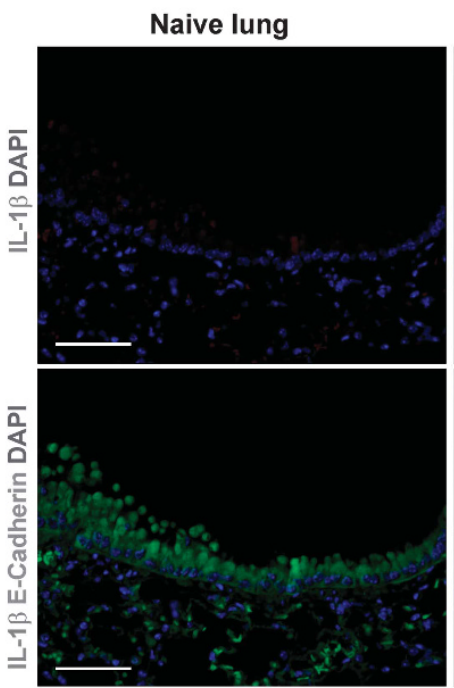

WT RSV 6 DPI
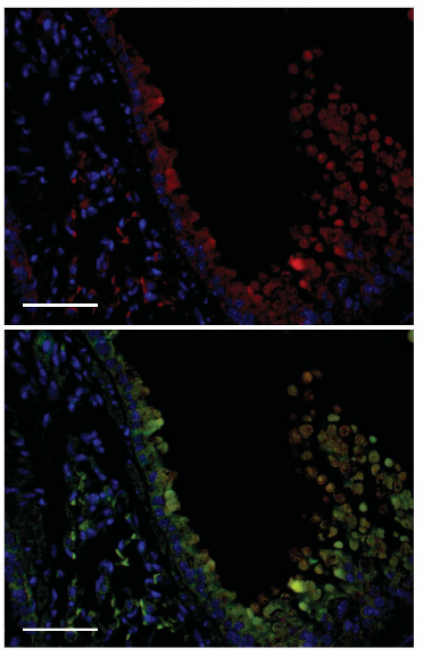

d IL-1 $\beta$

c

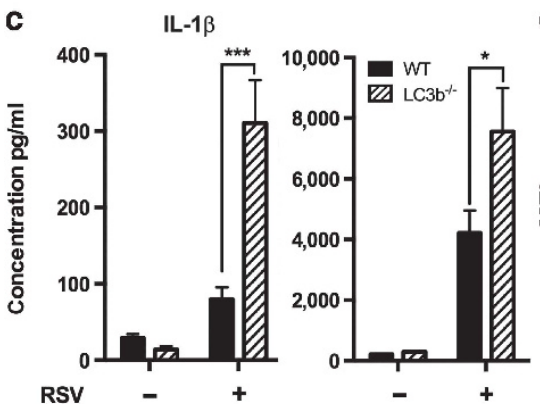

g

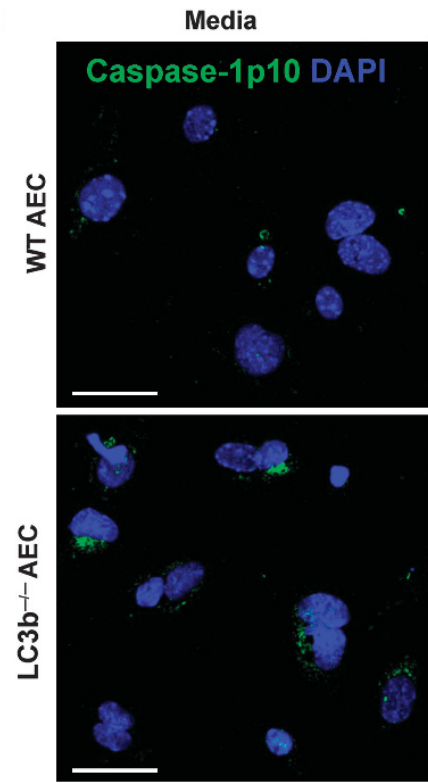

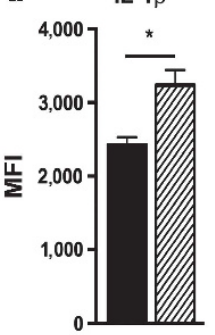

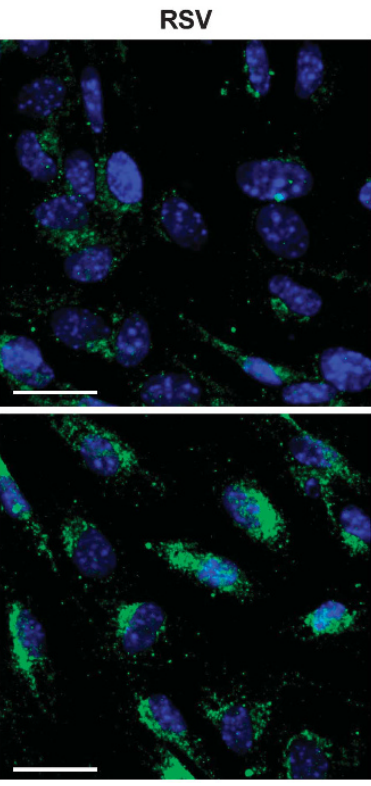

LC3b-1-RSV 6 DPI

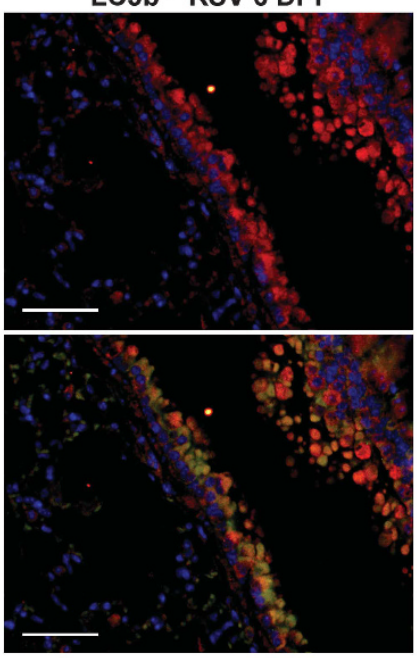

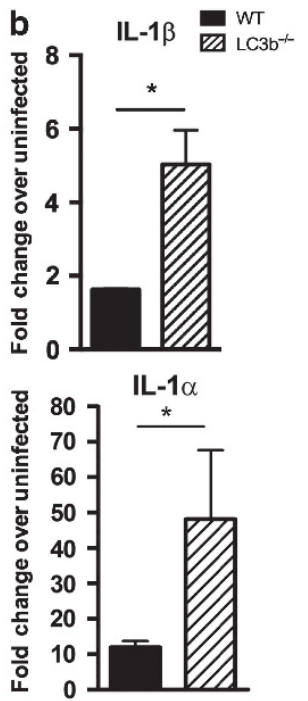
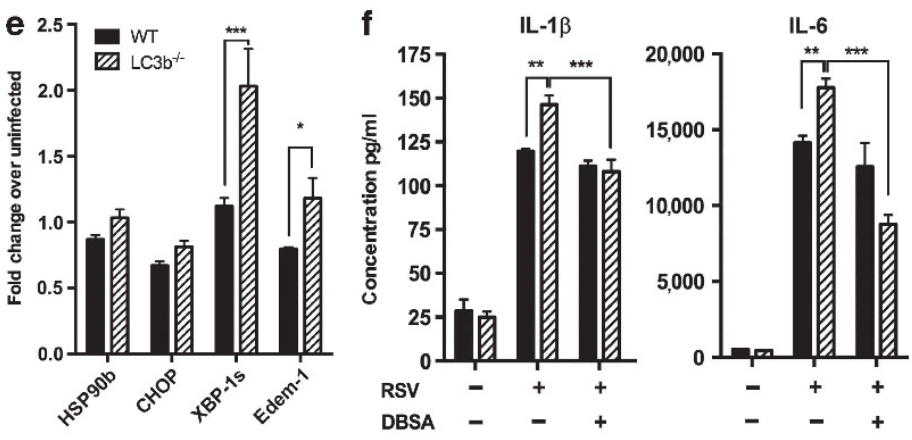

DBSA + RSV

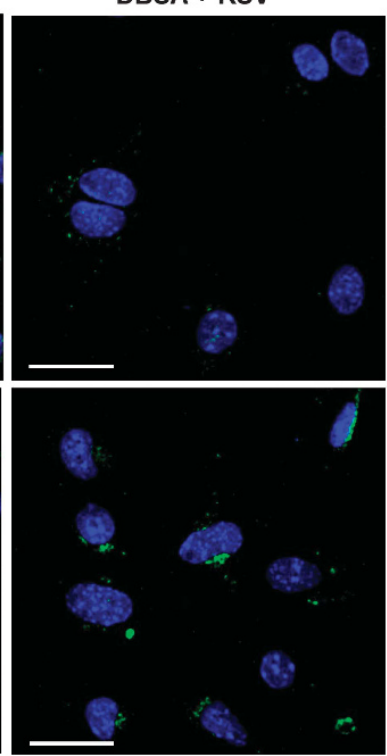

Caspase-1 p10

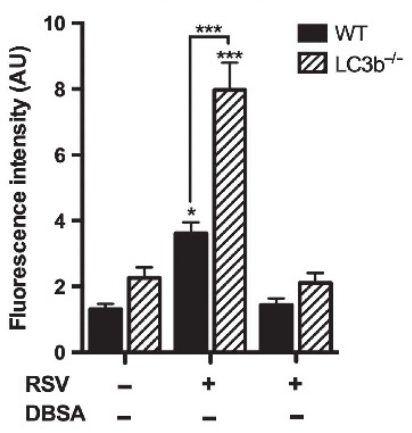

Figure 5 For caption see page 1126. 
epithelium is due to increased inflammasome activation and ER stress, and may have a critical role in shaping the ongoing immune response during RSV infection.

\section{Blockade of IL-1 receptor signaling ameliorates IL-17a-associated pathology in LC3b ${ }^{-/-}$mice in vivo}

In order to assess whether excessive IL-1 production was driving the development of the elevated Th17 response in
$\mathrm{LC} \mathrm{b}^{-/}$- mice, murine IL-1 receptor antagonist (IL-1Ra) was administered immediately before and during the course of RSV infection. Strikingly, $\mathrm{LC} 3 \mathrm{~b}^{-/-}$mice treated with IL-1Ra during RSV infection showed substantially reduced peribronchial inflammation in comparison with $\mathrm{LC} \mathrm{b}^{-/-}$saline treatment controls (Figure 6a). Treatment with IL-1Ra decreased periodic acid-schiff-positive mucus in the airways of RSV-infected $\mathrm{LC} \mathrm{b}^{-/-}$mice (Figure 6a, lower panels), a
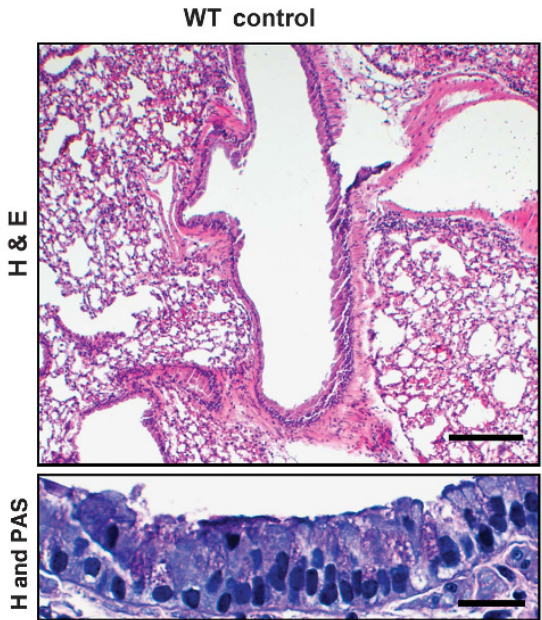
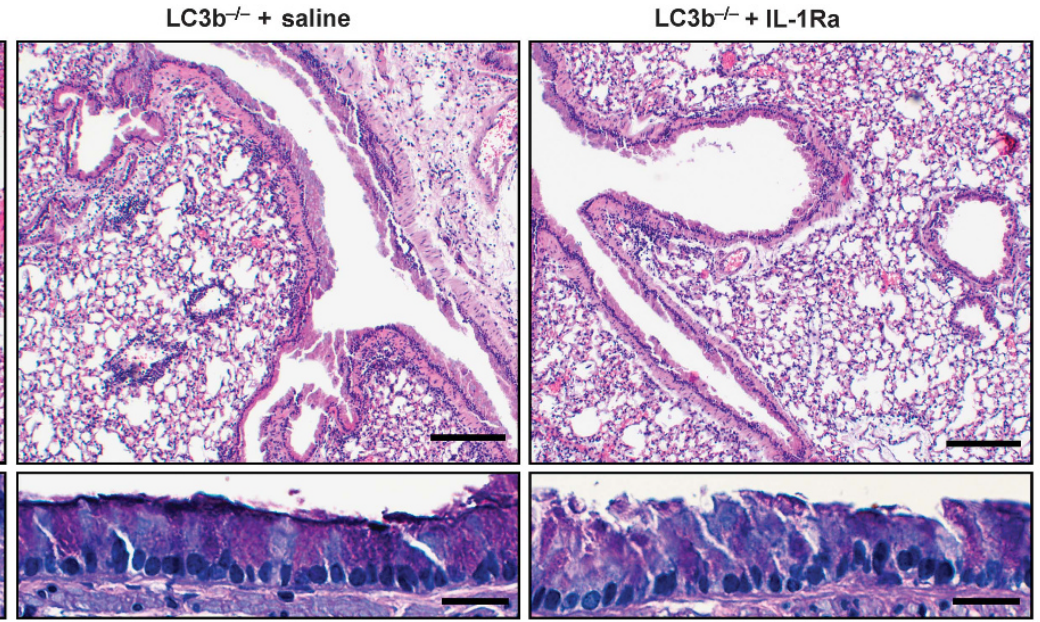

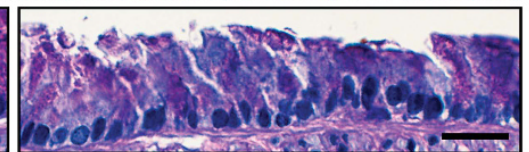

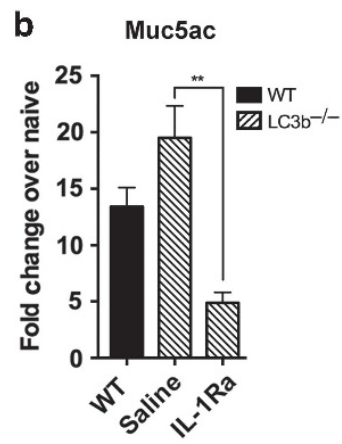
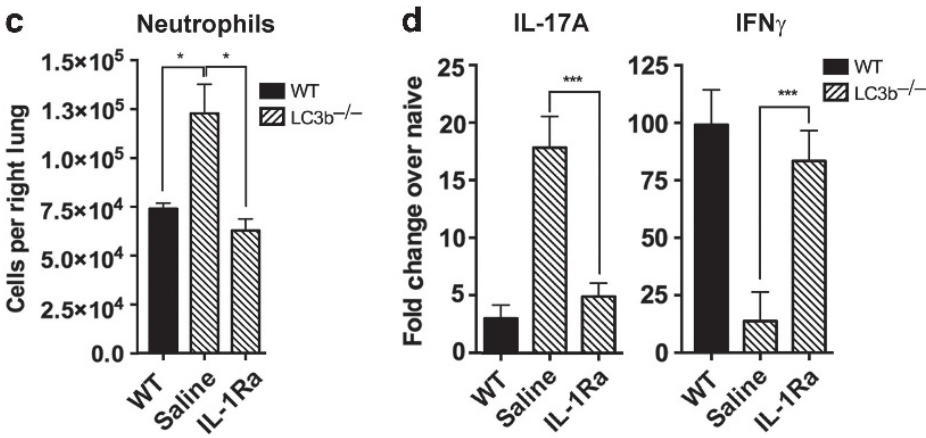

e

IL-17A

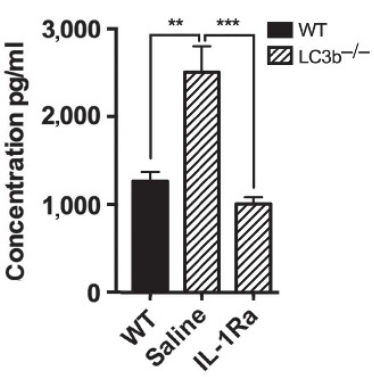

Figure 6 Increased interleukin (IL)-17a-dependent lung pathology in respiratory syncytial virus (RSV)-infected LC $3 b^{-/-}$mice is abrogated by blockade of IL-1 receptor signaling. LC3 $\mathrm{b}^{-1-}$ mice were injected intraperitoneally with $25 \mathrm{mg} / \mathrm{kg}$ mouse IL-1 receptor antagonist (IL-1Ra) immediately before RSV infection, and then treated once daily until being killed at 8 days post infection. (a) Lung sections were stained with hematoxylin and eosin (H \& E) or hematoxylin and periodic acid schiff (H and periodic acid-schiff (PAS)) to visualize mucus. (b) Lung expression of muc5ac was measured with quantitative PCR (qPCR). (c) Neutrophils were quantified in enzymatically digested lung tissue with flow cytometry, using cell surface antigens detailed in Figure 1. (d) Expression of IL-17a and interferon $\gamma$ (IFN $\gamma$ ) were measured in lungs using qPCR. (e) Single-cell suspensions prepared from mediastinal lymph nodes were restimulated in culture with RSV. IL-17a concentrations in supernatants were measured at $48 \mathrm{~h}$ post infection using the Bioplex assay. Results are representative of two independent experiments, $n \geq 5$ mice per group. ${ }^{\star} P \leq 0.05,{ }^{\star \star} P \leq 0.01,{ }^{\star \star \star} P \leq 0.001$. Bars $=100 \mu \mathrm{m}$ on upper panels, $20 \mu \mathrm{m}$ on lower panels.

Figure 5 Increased pro-inflammatory cytokine production by LC3b ${ }^{-1-}$ airway epithelial cells and lung macrophages upon respiratory syncytial virus (RSV) infection. (a) Lung sections from wild-type (WT) and LC3b ${ }^{-1-}$ mice, 6 days post infection, were stained with antibodies to mouse interleukin (IL)-1 $\beta$ and E-cadherin. Sections were counterstained with 4',6-diamidino-2-phenylindole (DAPI) and imaged on an Olympus BX43 fluorescent microscope. (b and $\mathbf{c}$ ) Airway epithelial cell (AEC) cultures were prepared from lungs of naive WT or LC3b ${ }^{-1-}$ mice, and infected with 1:1 multiplicity of infection (MOI) RSV. (b) IL- $1 \alpha$ and IL-1 $\beta$ expression was measured at $12 \mathrm{~h}$ post infection by quantitative PCR (qPCR), whereas (c) IL- $1 \beta$ and IL- 6 secretion was measured in culture supernatants at $24 \mathrm{~h}$ using the Bioplex assay. (d) Single-cell suspensions prepared from enzymatically digested lungs were surface-stained, followed by intracellular IL-1 $\beta$ staining, and analyzed using flow cytometry. Lung macrophages were gated Auto ${ }^{\text {hi }} \mathrm{CD} 11 \mathrm{~b}^{\text {hi }} \mathrm{F} 4 / 80^{+}$. Dark histograms represent isotype control antibodies, light histograms anti-IL-1 $\beta$ antibody. (e) Quantification of the median fluorescence intensity staining of lung macrophages for IL-1 3 . (e) mRNA transcripts of endoplasmic reticulum (ER) stress-associated genes HSP90, CHOP, Edem-1, and spliced XBP-1 were assessed in AECs at $12 \mathrm{~h}$ post infection using qPCR. (f) AECs were treated with the inositol-requiring protein-1 (IRE-1) $\alpha$ inhibitor 3,5-dibromosalicylaldehyde (DBSA) at $40 \mu \mathrm{M}$, or the general ER stress inhibitor 4-phenlbutyrate (4-PBA) at $5 \mathrm{mM}, 30 \mathrm{~min}$ before 1:1 MOI RSV. Cell culture supernatants were assayed at $24 \mathrm{~h}$ post infection using the Bioplex assay. (g) AECs were infected for $12 \mathrm{~h}$ with 1:1 MOI RSV, and then fixed and stained with antibodies to the Caspase-1 p10 subunit and DAPI. Cells were imaged on a Nikon A1 confocal microscope. Fluorescence intensity per cell was measured and quantified using maximum intensity projections, using the ImageJ software. Scale bars $=30 \mu \mathrm{m}$. Results are representative of at least two independent experiments. ${ }^{*} P \leq 0.05,{ }^{\star \star} P \leq 0.01$, ${ }_{* \star \star} P \leq 0.001$. Bars $=40 \mu \mathrm{m}$. 
a

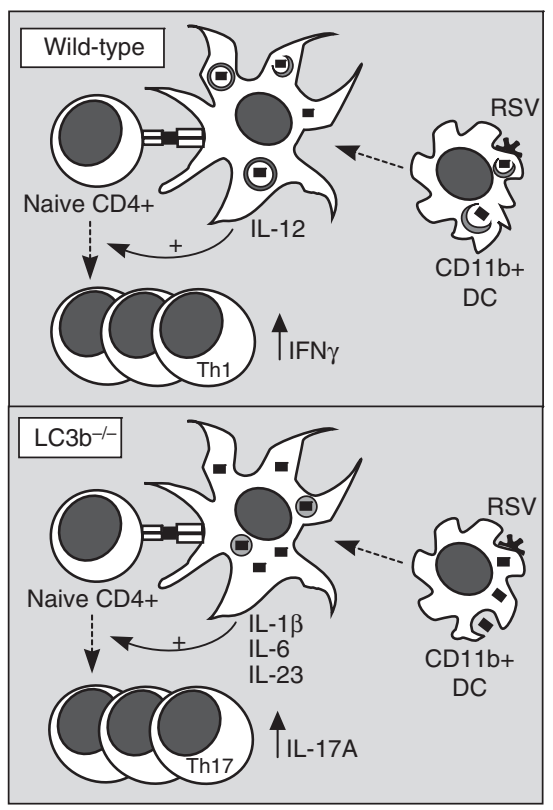

b

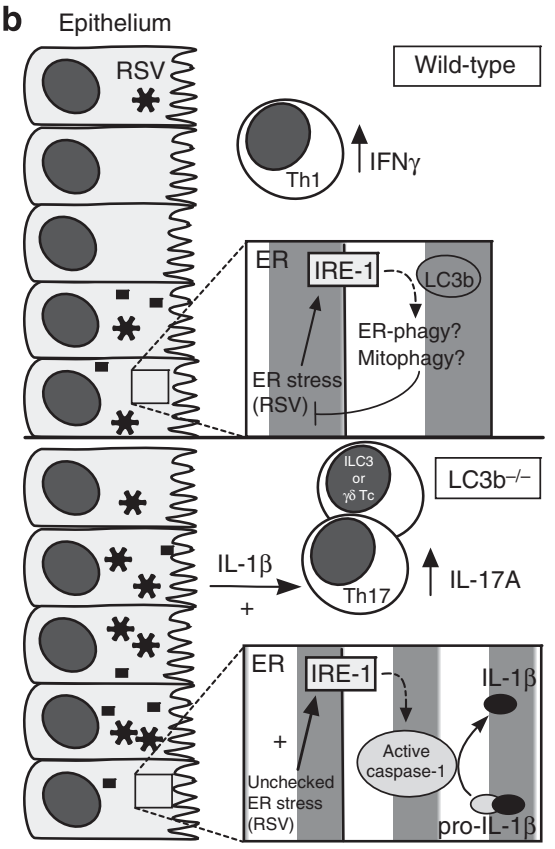

Figure 7 Proposed mechanisms of the induction and maintenance of CD4 + T-cell responses to respiratory syncytial virus (RSV) in wild-type or LC3b ${ }^{-\prime-}$ mice. (a) Upon detection of RSV by wild-type (WT) lung dendritic cells (DCs), rapid induction of autophagy facilitates interleukin (IL)-12 production, ultimately promoting the development of a Th1 response by RSV-reactive CD4 + T cells. In contrast, LC3b ${ }^{-1-}$ DCs fail to upregulate autophagy upon encountering RSV, resulting in the production of IL-1 $\beta$, IL-6, and IL-23. This cytokine environment skews RSV-reactive CD4 + T cells toward a Th17 phenotype. (b) RSV-infected lung epithelial cells upregulate signaling through the endoplasmic reticulum (ER) stress sensor inositol-requiring protein-1 (IRE-1), potentially triggering LC3b-dependent autophagosomal degradation of ER and damaged mitochondria, thereby alleviating RSV-induced stress and minimizing IL-1 $\beta$ production. In the absence of LC3b expression, RSV-infected epithelial cells experience increased IRE-1 signaling owing to unchecked ER stress, resulting in caspase- 1 cleavage and mature IL- $1 \beta$ production. This enhanced IL- $1 \beta$ secretion stimulates further IL-17a production from CD4 + effector T cells through IL-1 receptor signaling.

which was verified through significantly reduced mRNA expression of muc5ac in lung homogenates (Figure 6b). Quantification of lung neutrophils by flow cytometry revealed decreased neutrophil infiltration in IL-1Ra-treated LC3b ${ }^{-1-}$ mice, in comparison with saline-treated controls (Figure 6c). Examination of cytokine production in lungs using $\mathrm{qPCR}$ revealed significantly reduced IL-17a expression in IL-1Ratreated $\mathrm{LC} 3 \mathrm{~b}^{-1-}$ mice, with a concomitant increase in IFN $\gamma$ production in response to RSV infection (Figure 6d). Finally, IL-17a secretion by MedLN cells restimulated ex vivo was significantly reduced in cultures from $\mathrm{LC} \mathrm{b}^{-1-}$ mice that received IL-1Ra treatment (Figure 6e). These results ultimately suggest that elevated IL-17a production and associated lung pathology in RSV-infected LC3b ${ }^{-1-}$ mice are driven by IL-1 receptor signaling.

\section{DISCUSSION}

In this manuscript, we provide evidence for the importance of the autophagy protein LC3b in both innate cytokine production and the induction of adaptive immune responses to RSV. Mice deficient in LC3b developed greater IL-17a-dependent lung pathology upon RSV infection, characterized by neutrophil infiltration, mucus hypersecretion, and decreased viral clearance in comparison with WT littermates. The identification of $\mathrm{CD} 4+\mathrm{T}$ cells as the predominant IL-17a-positive cell type in
LC $3 \mathrm{~b}^{-1-}$ lungs at 8 days post-RSV infection concurs with previous studies in WT mice, ${ }^{10}$ and implicates enhanced DC-mediated induction of Th17 responses in the subsequent development of lung pathology. In agreement with previous work showing a requirement for autophagy in innate cytokine production and antigen presentation by DCs, ${ }^{18,24} \mathrm{RSV}$-infected $\mathrm{LC} \mathrm{b}^{-1-}$ DCs produced IL-1 $\beta$, IL-6, and IL-23 at the expense of IL-12 and preferentially elicited IL-17a production from $\mathrm{CD} 4+\mathrm{T}$ cells (Figure 7a). This preferential induction of Th17 responses to RSV was further evidenced by the development of elevated lung pathology in WT chimeric mice reconstituted with the $\mathrm{LC} \mathrm{b}^{-1-}$ bone marrow. However, $\mathrm{LC} 3 \mathrm{~b}^{-1-}$ chimeras reconstituted with WT bone marrow developed an augmented Th17 response to RSV, possibly because of both a robust induction of CD4 + T-cell responses by WT DCs, with elevated IL-17a production occurring in response to the highIL-1 environment of LC3b ${ }^{-1-}$ lungs (Figure $7 \mathbf{b}$ ). Indeed, infected LC3b ${ }^{-1-}$ AECs secreted greater amounts of IL- $1 \beta$ in a manner dependent on elevated IRE- $1 \alpha$ signaling. Finally, treatment of $\mathrm{LC} 3 \mathrm{~b}^{-1-}$ mice with IL-1Ra attenuated IL-17a production and associated lung pathology in response to RSV, implicating enhanced IL-1 receptor signaling in the development of IL-17-driven lung pathology. The identification of these innate pathways may lead to new strategies to alleviate pathophysiologic changes during RSV infection that 
lessen severity and subsequent long-term sequelae of pulmonary damage associated with RSV.

Since the discovery of Th17 cell lineage, aberrant or excessive Th17 immune responses have been found to enhance pathology in the context of immune-mediated disorders such as rheumatoid arthritis ${ }^{34}$ and allergic asthma, ${ }^{35}$ as well as during viral infection. ${ }^{36}$ Studies of severe RSV infection suggest that a Th17-skewed response contributes to lung pathology in at least a subset of patients, as elevated IL-17a expression has been reported in tracheal aspirates of infants hospitalized with RSV. ${ }^{10,37}$ In addition, lung expression levels of IL- 6 and IL- 1 are correlated with the severity of disease in both infants and adults infected with RSV., ${ }^{9,38}$ In the present studies, treatment of LC3 $b^{-1-}$ mice with anti-IL-17a antibodies during RSV infection significantly decreased lung neutrophil infiltration and mucus hypersecretion, while also elevating lung IFN $\gamma$ expression and restoring viral clearance. IL-17a may drive lung pathology by inducing mucus gene expression, ${ }^{39}$ potentiating IL-8 production in RSV-infected bronchial epithelial cells, thereby augmenting lung neutrophil recruitment, ${ }^{37}$ or by suppressing cytotoxic $\mathrm{CD} 8+\mathrm{T}$-cell activity ${ }^{10}$ and prolonging infection. This is supported by previous findings in our laboratory, as administration of neutralizing antibodies to IL-17a attenuated lung mucus production and neutrophil infiltration. ${ }^{10,28}$ The recovery of IFN $\gamma$ production in LC $3 \mathrm{~b}^{-1-}$ mice treated with anti-IL-17a antibodies is also likely because of a reduction in IL-17a, as IL-17a also suppresses the production of IFN $\gamma$ and cytotoxic molecules by CD8 + T cells in vivo. ${ }^{10}$ Moreover, in vivo blockade of IL-1 receptor signaling through IL-1Ra treatment mitigated the production of IL- 6 and IL-17a by the lung in RSV-infected $\mathrm{LC} \mathrm{b}^{-1-}$ mice, resulting in reduced lung pathology. While our data cannot rule out a lack of viral clearance as an explanation for increased pathology, the findings do provide further evidence in support of the critical role of LC3-dependent autophagy secretion during infectious insult ${ }^{26}$ as well as novel evidence supporting the role of autophagy in counteracting deleterious Th17 responses to RSV through blocking inflammasome-mediated production of IL-1 $\beta$.

The production of IL- 23 by DCs and macrophages is also indirectly regulated by autophagy, through autophagymediated inhibition of IL- 1 secretion. ${ }^{18}$ The alteration of autophagy reduces the ability to shuttle RSV RNA to endosomic TLR pathways, which have been shown to be associated with the regulation of IL-23 and IL-17a. ${ }^{28}$ Accordingly, we found that RSV-infected LC3b ${ }^{-1-}$ DCs that failed to upregulate autophagosome formation secreted more IL-1 $\beta$, IL-6, and IL-23, and preferentially induced IL-17a expression from co-cultured CD4 $+\mathrm{T}$ cells in comparison with WT controls. Experiments with chimeric mice further support a causative role for autophagy deficiency in DC-mediated induction of Th17 responses to RSV, as WT mice reconstituted with $\mathrm{LC} 3 \mathrm{~b}^{-1-}$ bone marrow developed similar lung pathology as mice fully deficient in LC3b. Conversely, the development of T-celldependent lung pathology in fully $\mathrm{LC}^{-1-}$ mice may be dampened not only by inefficient antigen presentation by autophagy-deficient DCs, ${ }^{40,41}$ but may also be because of a requirement for autophagy proteins in the survival and proliferation of effector T cells. ${ }^{42,43}$ In support of this, the reconstitution of $\mathrm{LC} \mathrm{b}^{-1-}$ mice with WT bone marrow augmented Th17-dependent lung pathology above levels observed in fully LC $3 \mathrm{~b}^{-1-}$ mice. Ultimately, these results suggest critical roles for LC3b-dependent autophagy in both structural and hematopoietic cells in mitigating inflammasome activation and the development of Th17-associated lung pathology during RSV infection.

Several nonexclusive mechanisms may contribute to increased IL- $1 \beta$ secretion by LC $3 \mathrm{~b}^{-1-}$ DCs, macrophages, and AECs in response to RSV infection. Our findings of increased IL- $1 \beta$ secretion and active caspase- 1 by $\mathrm{LC} \mathrm{b}^{-1-}$ AECs concur with two recent reports of RSV infection inducing IL-1 $\beta$ secretion in macrophages ${ }^{23}$ and primary human $\mathrm{AECs}^{22}$ through an NLRP3- and caspase-1-dependent mechanism. Although LC3b-dependent autophagy directly regulates inflammasome activity in macrophages through selective degradation of ubiquitinated NLRP3 inflammasome platforms ${ }^{21}$ and pro-IL-1 $\beta,{ }^{20}$ further work will be required to establish this mechanism in epithelial cells during RSV infection. The inability of $\mathrm{LC} \mathrm{b}^{-1-}$ cells to regulate inflammasome activation through autophagy may be further exacerbated by an inability to remove damaged mitochondria through mitophagy. ${ }^{26}$ Reactive oxygen species released by damaged mitochondria are required for NLRP3 inflammasome activation and caspase- 1 -mediated IL- $1 \beta$ production, ${ }^{44,45}$ and this was recently verified in RSV-infected macrophages. ${ }^{23}$ Autophagy-deficient $\mathrm{LC} \mathrm{b}^{-1-}$ macrophages accumulated damaged mitochondria and mitochondrial reactive oxygen species in response to lipopolysaccharide and ATP treatment, resulting in greater NLRP3 inflammasome activation and IL-1 $\beta$ secretion. ${ }^{26}$ As RSV infection was previously reported to increase numbers of morphologically altered mitochondria in human AECs ${ }^{46}$ an inability to sequester damaged mitochondria via LC3b-associated mechanisms may contribute to increased inflammasome activation upon infection.

Importantly, these data uncovered evidence of increased ER stress in LC3b ${ }^{-1-}$ AECs upon RSV infection. Recent data indicated that RSV infection of A549 cells activates ER stress response signaling through IRE-1 and ATF6. ${ }^{32}$ ER stress is known to upregulate pro-survival autophagy, ${ }^{47,48}$ whereas numerous studies have shown a requirement for IRE-1 pathway signaling in the regulation of autophagy during the ER stress response. ${ }^{47,49}$ It was also recently reported that the induction of ER stress activates the NLRP3 inflammasome and increases IL-1 $\beta$ secretion in a manner dependent on mitochondrial integrity. ${ }^{33}$ It is therefore plausible that ER stress in RSVinfected cells may serve the dual purpose of promoting cell survival through autophagy-mediated removal of viral nucleic acids and proteins, while simultaneously controlling NLRP3 inflammasome activation through the degradation of damaged mitochondria and assembled inflammasome platforms. Further studies will be required to elucidate the exact mechanisms underlying increased RSV-induced inflammasome activation and IL-1 $\beta$ secretion by $\mathrm{LC} \mathrm{b}^{-1-}$ cells. 
These findings provide additional evidence for the role of autophagy during RSV infection in promoting the induction of antiviral Th1 responses to RSV in vivo. Furthermore, these results suggest that LC3b-associated responses and autophagy serve as a critical negative regulator of inflammation during RSV infection, through the limitation of IL- $1 \beta$ production by lung epithelial cells. The present studies highlight the unique, cell-specific contribution of autophagy proteins to inflammatory processes, and provide valuable insight into the induction and development of Th17-skewed responses during RSV infection. Ultimately, this information may inform novel treatments for severe RSV infection, and ultimately aid in vaccine design strategies.

\section{METHODS}

Mice. Female C57Bl/6J and B6.Cg-Tg(TcraTcrb)425Cbn/J (OT-II) mice were purchased from The Jackson Laboratory (Bar Harbor, ME). B6;129P2-Map1lc3b tm1Mrab $/ \mathrm{J}$ were obtained from The Jackson Laboratory and were repeatedly backcrossed to $\mathrm{C} 57 \mathrm{Bl} / 6 \mathrm{~J}$ mice to produce WT or LC3b-deficient (LC3b ${ }^{-1-}$ ) mice of $\geq 98 \% \mathrm{C} 57 \mathrm{Bl} / 6$ background. All in vivo experiments utilized age- and sex-matched WT or LC3b ${ }^{-1-}$ littermates.

Bone marrow chimeras were created by irradiating WT or $\mathrm{LC}^{-1-}$ littermates at $900 \mathrm{rad}$, followed by tail-vein injection of $2 \times 10^{6}$ wholebone marrow cells of the genotype indicated. Chimeras were allowed to reconstitute for 8 weeks before RSV infection. All work involving animals was conducted in accordance with the University of Michigan Committee on Use and Care of Animals policy.

Respiratory syncytial virus. All experiments utilized antigenic subgroup A, Line 19 strain RSV, originally isolated from a sick infant in the University of Michigan Health System. Viral stocks were maintained in Hep-2 cell cultures.

Dendritic cell isolation and culture. BMDCs were cultured in complete media as previously described, ${ }^{25}$ from whole bone marrow in the presence of $20 \mathrm{ng} / \mathrm{ml}$ granulocyte macrophage colony-stimulating factor (R\&D Systems, Minneapolis, MN). Lung-resident CD11b + and CD103 + DCs were isolated by enzymatic digestion of lungs of naive WT or LC3b ${ }^{-1-}$ mice as previously described. ${ }^{25}$ Briefly, minced lung tissue was incubated with $200 \mu \mathrm{g} / \mathrm{ml}$ Liberase TM (Roche Applied Science, Indianapolis, IN) and $200 \mathrm{U} / \mathrm{ml}$ DNase I (Sigma-Aldrich, St Louis, MO), drawn through an 18-gauge needle, and filtered through a $40-\mu \mathrm{m}$ mesh. Cells were positively selected for CD11c expression using anti-mouse $\mathrm{CD} 11 \mathrm{c}$ microbeads and magnetic column separation (Miltenyi Biotec, Auburn, CA), followed by staining with PE-labeled anti-CD11b and allophycocyanin-labeled anti-CD103 Abs (eBioscience, San Diego, CA). Cells were sorted on an iCyt Synergy 3200 fluorescence-activated cell sorter (Biotechnology Inc., San Jose, CA) (Champaign, IL), followed by overnight culture in complete media.

For DC-T-cell co-culture experiments, RSV-responsive CD4 + T cells were obtained from mediastinal lymph nodes of RSV-infected C57Bl/6J mice, 8 days post infection. CD4 + OT-II T cells were isolated from minced spleens of OT-II transgenic mice. Cells were purified using a magnetic column negative-selection protocol, yielding untouched CD $4+$ T cells (Miltenyi Biotec). CD $4+$ T cells were added to the culture at a 10:1 ratio to DCs. For RSV-reactive T-cell cocultures, DCs were infected with 1:1 multiplicity of infection RSV $2 \mathrm{~h}$ before the addition of T cells. For OT-II T cell co-cultures, DCs were treated with $200 \mu \mathrm{g} / \mathrm{ml}$ whole ovalbumin protein, or concurrently treated with ovalbumin and 1:1 MOI of RSV, for $2 \mathrm{~h}$ before the addition of T cells. Culture supernatants were harvested at $48 \mathrm{~h}$ and analyzed using a custom Bio-Rad Bioplex PRO kit on a Bio-Rad Bioplex 200 system, according to the manufacturer's instructions (Bio-Rad, Hercules, CA).
AEC culture. AEC cultures were prepared from lungs of naive mice by digestion in Dispase (BD Biosciences, San Jose, CA), followed by filtration through a $25-\mu \mathrm{m}$ nylon mesh. Immune cells were depleted using biotinylated anti-CD45 antibodies and streptavidin-conjugated Dynabeads (Thermo Fisher Scientific, Waltham, MA). Cells were plated on $10-\mathrm{cm}$ tissue culture dishes and adherence-purified in DMEM-based complete media, followed by 4 -day culture in fibronectin-coated wells.

Reagents. Recombinant mouse IL-1Ra was obtained from R\&D systems. Neutralizing antibodies to mouse IL-17a were generated and purified in-house, as described previously. ${ }^{10}$ The ER stress inhibitors 3,5-dibromosalicylaldehyde and 4-phenylbutyrate were purchased from Santa Cruz Biotechnology (Dallas, TX).

Quantitative PCR. Total RNA was isolated from cell cultures and lung tissue using TRIzol reagent, according to the manufacturer's instructions (Invitrogen, Grand Island, NY). RNA was reversetranscribed, and cytokine gene expression was assessed using TaqMan Gene Expression Assay primer/probe sets on an ABI Prism 7500 Sequence Detection System (Applied Biosystems, Foster City, CA). Custom primers were used to assess transcription levels of muc5ac, gob5, RSV-G, and $R S V-F$, as previously described. ${ }^{50}$ Fold change expression was calculated from gene expression values normalized to GAPDH, relative to the indicated control group.

Flow cytometry. Cells were isolated from the right lungs and MedLNs by digestion in $200 \mu \mathrm{g} / \mathrm{ml}$ Liberase TM (Roche Applied Science) and 200 U/ml DNase I (Sigma-Aldrich). Cells were stained with Live/Dead Fixable Yellow (Invitrogen), followed by fluorescent antibodies as indicated. Analysis was performed using the FlowJo software (TreeStar, Ashland, OR).

Immunofluorescence and confocal microscopy. BMDCs and AECs were cultured as described above, and plated in Labtek chamber slides (Thermo Fisher Scientific). After indicated treatment, cells were fixed in $4 \%$ paraformaldehyde, followed by blocking in DPBS containing $5 \%$ normal goat serum and $0.1 \%$ Tween-20. Cells were stained with DyLight 550-conjugated anti-ATG5 antibodies (Novus Biologicals, Littleton, CO), or rabbit anti-Caspase-1 p10 (Santa Cruz Biotechnologies) followed by AlexaFluor 488-conjugated goat anti-rabbit antibodies (Jackson ImmunoResearch, West Grove, PA). Slides were mounted in ProLong Gold antifade reagent plus $4^{\prime}, 6$-diamidino-2phenylindole, and imaged using $\times 40$ oil immersion objective on a Nikon A1 Confocal Microscope System using the NIS Elements software (Nikon Instruments, Melville, NY). Maximum intensity projection images were generated using the ImageJ software (National Institutes of Health, Bethesda, MD).

For fluorescent immunohistochemistry, formalin-fixed paraffinembedded lung sections were de-parafinized, rehydrated, and incubated overnight in antigen retrieval buffer $(0.01 \mathrm{M}$ Citric acid, $\mathrm{pH}$ 6.0). Slices were blocked in DPBS $+2 \%$ normal goat serum, followed by incubation with rabbit anti-mouse IL-1 $\beta$ (Novus Biologicals) and biotinylated anti-E-cadherin (BD Biosciences). After washing in blocking buffer, the slices were incubated with anti-rabbit Alexa Fluor 568 and streptavidin-conjugated Alexa Fluor 488 (BD Biosciences). Images were taken using a $\times 40$ objective on an Olympus BX43 fluorescent microscope.

Statistics. Data were analyzed and graphed using the GraphPad Prism software (San Diego, CA). Statistical significance was determined by oneway analysis of variance and Bonferroni post-test to obtain $P$-values.

\section{ACKNOWLEDGMENTS}

We acknowledge the editing assistance of Dr Judith Connett, technical assistance provided by $\mathrm{Dr}$ Matthew Schaller, and histological slide preparation by Lisa Riggs Johnson. We also thank the Flow Cytometry and Microscopy Imaging and Analysis Core Facilities at the University of Michigan. This work was supported by NIH-HL-114858. 


\section{DISCLOSURE}

The authors declare no conflict of interest.

(c) 2015 Society for Mucosal Immunology

\section{REFERENCES}

1. Leader, S. \& Kohlhase, K. Recent trends in severe respiratory syncytial virus (RSV) among US infants, 1997 to 2000. J. Pediatr. 143, S127-S132 (2003).

2. Stockman, L.J., Curns, A.T., Anderson, L.J. \& Fischer-Langley, G. Respiratory syncytial virus-associated hospitalizations among infants and young children in the United States, 1997-2006. Pediatr. Infect. Dis. J. 31, 5-9 (2012)

3. Falsey, A.R., Hennessey, P.A., Formica, M.A., Cox, C. \& Walsh, E.E. Respiratory syncytial virus infection in elderly and high-risk adults. N. Engl. J. Med. 352, 1749-1759 (2005).

4. Tan, W.C. Viruses in asthma exacerbations. Curr. Opin. Pulm. Med. 11 21-26 (2005).

5. Wedzicha, J.A. Role of viruses in exacerbations of chronic obstructive pulmonary disease. Proc. Am. Thorac. Soc. 1, 115-120 (2004).

6. Sigurs, N. et al. Severe respiratory syncytial virus bronchiolitis in infancy and asthma and allergy at age 13. Am. J. Respir. Crit. Care Med. 171, 137-141 (2005).

7. van Drunen Littel-van den Hurk, S. \& Watkiss, E.R. Pathogenesis of respiratory syncytial virus. Curr. Opin. Virol. 2, 300-305 (2012).

8. Johnson, J.E., Gonzales, R.A., Olson, S.J., Wright, P.F. \& Graham, B.S. The histopathology of fatal untreated human respiratory syncytial virus infection. Mod. Pathol. 20, 108-119 (2006).

9. Duncan, C.B., Walsh, E.E., Peterson, D.R., Lee, F. E.-H. \& Falsey, A.R. Risk factors for respiratory failure associated with respiratory syncytial virus infection in adults. J. Infect. Dis. 200, 1242-1246 (2009).

10. Mukherjee, S. et al. IL-17-induced pulmonary pathogenesis during respiratory viral infection and exacerbation of allergic disease. Am. J. Pathol. 179, 248-258 (2011).

11. Tabarani, C.M. et al. Novel inflammatory markers, clinical risk factors and virus type associated with severe respiratory syncytial virus infection. Pediatr. Infect. Dis. J. 32, e437-e442 (2013)

12. Bera, M.M. et al. Th17 cytokines are critical for respiratory syncytial virusassociated airway hyperreponsiveness through regulation by complement C3a and tachykinins. J. Immunol. 187, 4245-4255 (2011).

13. Ryzhakov, G. et al. IL-17 boosts proinflammatory outcome of antiviral response in human cells. J. Immunol. 187, 5357-5362 (2011).

14. Bettelli, E. et al. Reciprocal developmental pathways for the generation of pathogenic effector TH17 and regulatory T cells. Nature 441, 235-238 (2006).

15. Mangan, P.R. et al. Transforming growth factor-beta induces development of the $T(H) 17$ lineage. Nature 441, 231-234 (2006).

16. Acosta-Rodriguez, E.V., Napolitani, G., Lanzavecchia, A. \& Sallusto, F. Interleukins 1 beta and 6 but not transforming growth factor-beta are essential for the differentiation of interleukin 17-producing human Thelper cells. Nat. Immunol. 8, 942-949 (2007).

17. Hu, W., Troutman, T.D., Edukulla, R. \& Pasare, C. Priming microenvironments dictate cytokine requirements for $\mathrm{T}$ helper 17 cell lineage commitment. Immunity 35, 1010-1022 (2011).

18. de Castro, C.P. et al. Autophagy regulates IL-23 secretion and innate Tcell responses through effects on IL-1 secretion. J. Immunol. 189, 4144-4153 (2012).

19. Latz, E., Xiao, T.S. \& Stutz, A. Activation and regulation of the inflammasomes. Nat. Rev. Immunol. 13, 397-411 (2013).

20. Harris, J. et al. Autophagy controls IL-1 $\beta$ secretion by targeting pro-IL-1 $\beta$ for degradation. J. Biol. Chem. 286, 9587-9597 (2011).

21. Shi, C.-S. et al. Activation of autophagy by inflammatory signals limits IL-1 $\beta$ production by targeting ubiquitinated inflammasomes for destruction. Nat. Immunol. 13, 255-263 (2012).

22. Triantafilou, K., Kar, S., Vakakis, E., Kotecha, S. \& Triantafilou, M. Human respiratory syncytial virus viroporin $\mathrm{SH}$ : a viral recognition pathway used by the host to signal inflammasome activation. Thorax 68, 66-75 (2013).

23. Segovia, J. et al. TLR2/MyD88/NF-kB pathway, reactive oxygen species, potassium efflux activates NLRP3/ASC Inflammasome during respiratory syncytial virus infection. PLOS ONE 7, e29695 (2012).
24. Morris, S. et al. Autophagy-mediated dendritic cell activation is essential for innate cytokine production and APC function with respiratory syncytial virus responses. J. Immunol. 187, 3953-3961 (2011).

25. Reed, M. et al. Autophagy-inducing protein Beclin-1 in dendritic cells regulates CD4 T cell responses and disease severity during respiratory syncytial virus infection. J. Immunol. 191, 2526-2537 (2013).

26. Nakahira, K. et al. Autophagy proteins regulate innate immune responses by inhibiting the release of mitochondrial DNA mediated by the NALP3 inflammasome. Nat. Immunol. 12, 222-230 (2011).

27. Lukacs, N.W. et al. Respiratory virus-induced TLR7 activation controls IL17-associated increased mucus via IL-23 regulation. J. Immunol. 185, 2231-2239 (2010).

28. Schlitzer, A. et al. IRF4 transcription factor-dependent CD11b + dendritic cells in human and mouse control mucosal IL-17 cytokine responses. Immunity 38, 970-983 (2013).

29. Persson, E.K. et al. IRF4 transcription-factor-dependent CD103(+) $\mathrm{CD} 11 \mathrm{~b}(+)$ dendritic cells drive mucosal T helper 17 cell differentiation. Immunity 38, 958-969 (2013).

30. Chen, Z.-H. et al. Autophagy protein microtubule-associated protein 1 light chain-3B (LC3B) activates extrinsic apoptosis during cigarette smokeinduced emphysema. Proc. Natl Acad. Sci. USA 107, 18880-18885 (2010).

31. Tanaka, A. et al. Hyperoxia-induced LC3B interacts with the Fas apoptotic pathway in epithelial cell death. Am. J. Respir. Cell Mol. Biol. 46, 507-514 (2012).

32. Hassan, I. et al. The inositol requiring enzyme 1 inhibits respiratory syncytial virus replication. J. Biol. Chem. 289, 7537-7546 (2014).

33. Menu, P. et al. ER stress activates the NLRP3 inflammasome via an UPRindependent pathway. Cell Death Dis. 3, e261 (2012).

34. Bedoya, S.K., Lam, B., Lau, K. \& Larkin, J. 3rd Th17 cells in immunity and autoimmunity. Clin. Dev. Immunol. 2013, 986789 (2013).

35. Wang, Y.-H. \& Wills-Karp, M. The potential role of interleukin-17 in severe asthma. Curr. Allergy Asthma Rep. 11, 388-394 (2011).

36. Crowe, C.R. et al. Critical role of IL-17RA in immunopathology of influenza infection. J. Immunol. 183, 5301-5310 (2009).

37. Stoppelenburg, A.J. et al. Local IL-17A potentiates early neutrophil recruitment to the respiratory tract during severe RSV infection. PLoS One 8, e78461 (2013).

38. Faber, T.E., Groen, H., Welfing, M., Jansen, K.J.G. \& Bont, L.J. Specific increase in local IL-17 production during recovery from primary RSV bronchiolitis. J. Med. Virol. 84, 1084-1088 (2012).

39. Chen, Y. et al. Stimulation of airway mucin gene expression by interleukin (IL)-17 through IL-6 paracrine/autocrine loop. J. Biol. Chem. 278, 17036-17043 (2003).

40. English, L. et al. Autophagy enhances the presentation of endogenous viral antigens on $\mathrm{MHC}$ class I molecules during HSV-1 infection. Nat. Immunol. 10, 480-487 (2009).

41. Chemali, M., Radtke, K., Desjardins, M. \& English, L. Alternative pathways for $\mathrm{MHC}$ class I presentation: a new function for autophagy. Cell. Mol. Life Sci. 68, 1533-1541 (2011).

42. Pua, H.H., Dzhagalov, I., Chuck, M., Mizushima, N. \& He, Y.-W. A critical role for the autophagy gene Atg5 in T cell survival and proliferation. J. Exp. Med. 204, 25-31 (2007).

43. Pua, H.H., Guo, J., Komatsu, M. \& He, Y.-W. Autophagy is essential for mitochondrial clearance in mature T lymphocytes. J. Immunol. 182, 4046-4055 (2009).

44. Heid, M.E. et al. Mitochondrial reactive oxygen species induces NLRP3dependent lysosomal damage and inflammasome activation. J. Immunol. 191, 5230-5238 (2013).

45. Zhou, R., Yazdi, A.S., Menu, P. \& Tschopp, J. A role for mitochondria in NLRP3 inflammasome activation. Nature 469, 221-225 (2011)

46. Smith, C.M. et al. Ciliary dyskinesia is an early feature of respiratory syncytial virus infection. Eur. Respir. J. 43, 485-496 (2013).

47. Ogata, M. et al. Autophagy is activated for cell survival after endoplasmic reticulum stress. Mol. Cell Biol. 26, 9220-9231 (2006).

48. Yorimitsu, T., Nair, U., Yang, Z. \& Klionsky, D.J. Endoplasmic reticulum stress triggers autophagy. J. Biol. Chem. 281, 30299-30304 (2006).

49. Castillo, K. et al. BAX inhibitor-1 regulates autophagy by controlling the IRE $1 \alpha$ branch of the unfolded protein response. EMBO J. 30, 4465-4478 (2011).

50. Miller, A.L., Bowlin, T.L. \& Lukacs, N.W. Respiratory syncytial virus-induced chemokine production: linking viral replication to chemokine production in vitro and in vivo. J. Infect. Dis. 189, 1419-1430 (2004). 